\title{
Thermoelectric effects in graphene nanostructures
}

\author{
Philippe Dollfus ${ }^{1}$, Viet Hung Nguyen and Jérôme Saint-Martin \\ Institut d'Electronique Fondamentale (IEF), Université Paris-Sud, CNRS, UMR 8622, \\ Orsay, France \\ E-mail: philippe.dollfus@u-psud.fr
}

\begin{abstract}
Thermoelectric properties of graphene and graphene nanostructures have recently attracted significant attention from the physics and engineering communities. In fundamental physics, the analysis of Seebeck and Nernst effects is very useful to elucidate some details of the electronic band structure of graphene that cannot be probed by conductance measurements alone, due in particular to the ambipolar nature of this gapless material. For applications in thermoelectric energy conversion, graphene has the two major disadvantages of being gapless, which leads to a small Seebeck coefficient due to the opposite contributions of electrons and holes, and to be an excellent thermal conductor. The thermoelectric figure of merit $Z T$ of a two-dimensional graphene sheet is thus very limited. However, many works have demonstrated recently that appropriate nanostructuring and bandgap engineering of graphene can concomitantly reduce strongly the lattice thermal conductance and enhance the Seebeck coefficient without dramatically degrading the electronic conductance. Hence, in various graphene nanostructures, $Z T$ has been predicted to be high enough to make them attractive for energy conversion. In this article, we review the main results obtained experimentally and theoretically on the thermoelectric properties of graphene and its nanostructures, emphasizing the physical effects that govern these properties. Beyond pure graphene structures, we discuss also the thermoelectric properties of some hybrid graphene structures, as graphane, layered carbon allotropes as graphynes and graphdiynes, and graphene / hexagonal boron nitride heterostructures which offer new opportunities. Finally, we briefly review the recent activities on other atomically thin 2D semiconductors with finite bandgap, i.e. dichalcogenides and phosphorene, which have attracted great attention for various kinds of applications, including thermoelectrics.
\end{abstract}

PACS Numbers: 72.20.Pa, 73.22.Pr, 73.23.Ad, 73.63.-b, 73.50.Lw, 85.80.Fi

\section{Introduction - Why graphene and graphene nanostructures for thermoelectrics?}

Thermoelectric effects refer to the direct conversion between a temperature difference and an electrical voltage. As for all heat engines, the efficiency of a thermoelectric system with hot end at temperature $T_{h}$ and cold end at temperature $T_{c}$ should reach the theoretical Carnot efficiency $\eta_{\text {Carnot }}=1-T_{c} / T_{h}$ if the thermoelectric cycle was fully reversible. Practically, it is strongly limited by the irreversible losses, i.e. the Joule heating and the thermal conduction, and by the thermoelectric properties of the material. The maximum efficiency writes

$$
\eta_{\max }=\eta_{\text {Carnot }} \times \frac{\sqrt{1+Z T}-1}{\sqrt{1+Z T}+\frac{T_{c}}{T_{h}}},
$$


where $Z T$ is a dimensionless parameter that measures the performance of the thermoelectric material. It is called thermoelectric figure of merit and is defined as

$$
Z T=\frac{\sigma S^{2} T}{\kappa}
$$

where $\sigma$ is the electrical conductivity, $S$ is the Seebeck coefficient (or thermoelectric power), $\kappa=\kappa_{e}+\kappa_{p}$ is the thermal conductivity including both electron and phonon contributions, and $T$ is the temperature. It is sometimes convenient to define $Z T$ in terms of ratio of electrical and thermal conductances $\mathrm{G}$ and $K$ instead of conductivities $\sigma$ and $\kappa$. For the practical viability of thermoelectric applications, designing materials with high $Z T$ is obviously the main issue in thermoelectric material research. Unfortunately, in bulk materials each factors entering the expression of $Z T$ are mutually coupled in such a way that it is very difficult to control them independently, and thus to improve $Z T$. Indeed, according to the Wiedemann-Franz law, increasing $\sigma$ leads to an increase in the electronic contribution to $\kappa$, and the Mott formula tells us that an increase in $\sigma$ tends to result in a decrease in $S$. Hence, to enhance $Z T$, the best strategy is often to minimize the lattice thermal conductivity, e.g. by introducing heavy atoms, disorder or clusters likely to increase the phonon scattering without degrading drastically the electronic conductivity. However, increasing the power factor $\sigma S^{2}$ may be a good option too, in particular in the case of graphene that offers a very high electrical conductivity.

It is important to have in mind some basic principles on the choice of materials suitable for thermoelectrics. A large Seebeck coefficient is usually found in low carrier concentration semiconductors while a large electrical conductivity is found in high carrier concentration metals. To maximize the power factor $\sigma S^{2}$, the best compromise is to use heavily-doped semiconductors. Indeed, in a semiconductor with a finite bandgap, electrons and holes can be separated and doping produces a high density of a single carrier type, so that the Seebeck coefficient is not reduced by opposite contributions of electrons and holes. Thus good thermoelectric materials have a bandgap large enough to have only a single type of carrier but small enough to make high doping level possible, to achieve high electrical conductivity.

Among the materials selected to offer the best compromise, $\mathrm{PbTe}, \mathrm{Bi}_{2} \mathrm{Te}_{3}$ and their alloys were found in the 1950s to provide high values of $Z T$ [1][2], which motivated the creation of the thermoelectric industry. However, over the following three decades, only small gains were achieved in increasing $Z T$, to such a point that the thermoelectrics community tended to consider the value of about 1 as an upper limit for $Z T$. If we except the progress made in skutterudite materials [3][4] and in HalfHeusler alloys [5][6], the best thermoelectric material was found in the $\left(\mathrm{Bi}_{1-\mathrm{x}} \mathrm{Sb}_{\mathrm{x}}\right)_{2}\left(\mathrm{Se}_{1-\mathrm{y}} \mathrm{Te}_{\mathrm{y}}\right)_{3}$ alloy family, with $Z T \approx 1$ at room temperature. This class of commercially available materials currently dominates in temperature control and thermal management applications. High temperature materials such as silicon-germanium ( $\mathrm{SiGe}$ ) alloys are heavily used for space exploration [7]. Actually, until now the slow growth of the thermoelectrics industry was based only on niche applications for which the cost and the energy efficiency are not important issues. A value of $Z T \approx 3$ is desirable to broaden the use of thermoelectric solid-state devices by making them competitive with conventional mechanical energy conversion systems.

A renewal of the thermoelectrics material research came in the 1990s from the pioneering works of Hicks and Dresselhaus [8] suggesting that nanostructuring materials into two-dimensional (2D) systems should provide much higher $Z T$ (>1) and thermoelectric efficiencies than with bulk materials. They even predicted still higher $Z T$ in one-dimensional (1D) nanowires [9]. This trend has been confirmed by many experiments and further theoretical investigations. This enhancement of $Z T$ 
in low-dimensional systems has two main origins, i.e. (i) the Seebeck coefficient enhancement resulting from size-quantization and (ii) the reduction of phonon thermal conductivity due to interface effects.

The size-quantization effect may be understood as follows. For a given conductor, an energydependent conductivity $\sigma(E)$ can be associated to the electrons of density $n(E)$ that fill the energy states between $E$ and $E+d E$. It writes

$$
\sigma(E)=e n(E) \mu(E) \text {, }
$$

where $e$ is the free electron charge and $\mu(E)$ is the mobility. For a conductor of length $L$ and section $S$, the total conductivity can be derived as [10]

$$
\sigma=G \frac{L}{S}=\int \sigma(E)\left(-\frac{\partial f(E)}{\partial E}\right) d E,
$$

where $f(E)$ is the Fermi distribution function. It should be noted that for very small conductors, it may be more relevant to consider the conductance $G$ that can be formulated in the Landauer formalism in terms of energy-dependent transmission $T(E)$ as

$$
G=\frac{2 e^{2}}{h} \int T(E)\left(-\frac{\partial f(E)}{\partial E}\right) d E .
$$

In the same formalism as for Eq. (4), Cutler and Mott derived the Seebeck coefficient in the form known as the Mott relation as [11]

$$
S=\frac{1}{\sigma} \frac{k_{B}}{e} \int \sigma(E)\left(\frac{E-E_{F}}{k_{B} T}\right)\left(-\frac{\partial f(E)}{\partial E}\right) d E .
$$

In degenerately doped systems, Eq. (6) simplifies into the well-known form

$$
S=\frac{\pi^{2}}{3} \frac{k_{B}}{e} k_{B} T\left[\frac{d \ln (\sigma(E))}{d E}\right]_{E=E_{F}} .
$$

Hence, to enhance the Seebeck coefficient $S$, it is clear that any effect likely to enhance the energydependence of the conductivity $d \sigma(E) / d E$, e.g. by enhancing the energy-dependence of the density $n(E)$, which is directly dependent on the density of states $g(E)$. Compared to 3D systems with typically a square root energy-dependence of $g(E)$, it is clear that 2D systems and especially 1D and OD systems provide much higher $d g(E) / d E$ that should be reflected on $S$. It was even shown that the shape of density of states that maximizes the power factor $\sigma S^{2}$ and $Z T$ is a delta function of energy [12]. Experimentally, this effect of size quantization on the enhancement of $\sigma S^{2}$ has been reported first in $\mathrm{PbTe} / \mathrm{Pb}_{1-\mathrm{x}} \mathrm{Eu}_{\mathrm{x}} \mathrm{Te}$ quantum well structures [13].

The second important effect expected from low-dimensional systems is related to interfaces which scatter phonons more effectively than charge carriers, and particularly phonons that contribute strongly to the lattice thermal conductivity. In superlattices, the impact of interfaces on thermal conductivity may have various origins, including bandgap formation, phonon localization [14], specular scattering due to acoustic mismatch [15], and scattering at dislocations [16]. This is expected to result in a reduction of thermal conductivity stronger than the concomitant reduction of electrical conductivity due to lower mobility. To minimize the latter reduction, choosing a couple of material with very small band offsets, as $\mathrm{Bi}_{2} \mathrm{Te}_{3} / \mathrm{Sb}_{2} \mathrm{Te}_{3}$, is a good option. Actually, in superlattices this effect of reduction of lattice thermal conductivity in cross-plane direction has been shown to yield a greater contribution to 
the enhancement of $Z T$ than the effect of power factor enhancement due to size-quantization. A wide range of material systems has been explored to assess the effect of superlattice structuring on thermoelectric properties, including $\mathrm{PbTe} / \mathrm{Te}$ [17], $\mathrm{PbTe} / \mathrm{PbSe}_{\mathrm{x}} \mathrm{Te}_{1-\mathrm{x}}$ [18][19], $\mathrm{Si} / \mathrm{Si}_{1-\mathrm{x}} \mathrm{Ge}_{\mathrm{x}}$ [20], GaAs/ $\mathrm{Ga}_{1-\mathrm{x}} \mathrm{Al}_{\mathrm{x}} \mathrm{As}$ [21], $\mathrm{Ga}_{1-\mathrm{x}} \mathrm{In}_{\mathrm{x}} \mathrm{As} / \mathrm{Ga}_{-\mathrm{y}-\mathrm{z}} \mathrm{Al}_{\mathrm{y}} \mathrm{In}_{\mathrm{z}} \mathrm{As}$ [22], and $\mathrm{Bi}_{2} \mathrm{Te}_{3} / \mathrm{Sb}_{2} \mathrm{Te}_{3}$ [23]. Today, it is usually accepted that the best $Z T$ results were achieved in $\mathrm{PbTe} / \mathrm{PbSe}_{\mathrm{x}} \mathrm{Te}_{1-\mathrm{x}}$ [18], with $Z T=1.6$ at room temperature and 3.5 at $570 \mathrm{~K}$, in $\mathrm{Bi}_{2} \mathrm{Te}_{3} / \mathrm{Sb}_{2} \mathrm{Te}_{3}$ [23], with $Z T=2.4$ at room temperature, and even in SnSe without nanostructuring [24], with $Z T=2.6$ at $T=923 \mathrm{~K}$. However, it should mentioned that some experimental results obtained for the Seebeck coefficient and the figure of merit are still controversial (see e.g. the discussion in Refs. [25] and [26]).

Further improvements were naturally searched in nanowire (NW) and quantum dot (QD) structures, in particular to benefit from higher size-quantization effects, with the possibility to include superlattices and quantum dots along nanowires [27]. For a long time, it was difficult to demonstrate high values of $Z T$ in nanowire thermoelectric devices. Indeed, for practical applications, nanowire arrays are required and should be embedded in a matrix, with difficulties in ensuring good electrical and thermal contact to all nanowires and to minimize the matrix-induced heat leakage. However, though bulk $\mathrm{Si}$ is a poor thermoelectric material due to its high thermal conductivity, it has been shown that in rough $\mathrm{Si}$ nanowires $Z T$ can reach the value of 0.6 at room temperature (and about 1 at $200 \mathrm{~K}$ ) thanks to a huge reduction of the thermal conductivity approaching the amorphous limit for $\mathrm{Si}$, while the Seebeck coefficient and the electrical conductivity are the same as in bulk-Si [28][29]. Recently, a thermoelectric generator consisting of a $\mathrm{Cu} / 20 \mu \mathrm{m}-\mathrm{Si}_{0.8} \mathrm{Ge}_{0.2} \mathrm{NW}$ array/bulk-Si/Cu structure has been shown to increase the output power by a factor of 20 with respect to its bulk-Si counterpart, thanks to reduced thermal conductivity and contact resistance [30]. Finally, theoretical investigations based on accurate first-principles and non-equilibrium Green's function simulation have predicted that in $\mathrm{Si} / \mathrm{Ge}$ superlattice nanowires, $Z T$ can be 5 times higher than in Si NWs, reaching 4.7 with n-type NWs and 2.7 with p-type NWs [31]. It was again attributed to a strong reduction in lattice thermal conductance that largely offsets the decrease in power factor.

Many superlattice (SL) systems made of three-dimensional arrays of quantum dots (QD) grown via Stranski-Krastanov or Volmer-Weber mechanisms have been also investigated for thermoelectric applications. We should mention in particular the case of systems based on traditional $\mathrm{Pb}-\mathrm{Se}-\mathrm{Te}$ thermoelectric materials [32][33][34][35] and also on Ge dots embedded in an Si matrix [36]. In $\mathrm{PbTe} / \mathrm{Pb}_{1-y} \mathrm{Sn}_{\mathrm{y}} \mathrm{Te}_{1-\mathrm{x}} \mathrm{Se}_{\mathrm{x}}$ QDSLs a value $Z T=2$ has been reported at room temperature, which is about four times higher than $Z T$ of bulk PbTe [32]. This enhancement is due to both a strong decrease in thermal conductivity and an increase in the Seebeck coefficient that offsets the reduction in mobility. Similar effects may be obtained from the inclusion of nanoprecipitates in bulk PbTe. It was shown that in $\mathrm{PbTe}$ with $\mathrm{Pb}$ precipitates, the enhancement of Seebeck coefficient is due to an increase in the energy-dependence of the relaxation time [37].

For in-depth description and discussion of thermoelectrics at the nanoscale, the reader should refer to some review articles dedicated to low-dimensional thermoelectric materials e.g. by Heremans [38], Dresselhaus et al. [7], Vineis et al. [25] and Liu et al. [39].

Now we come to the specific case of graphene. This material consists of a single layer of carbon atoms, arranged in a honeycomb lattice. The covalent bonds between nearest-neighbour atoms are formed by $\mathrm{sp}^{2}$-hybridized states. These strong bonds give graphene its extraordinary mechanical strength, stable enough to make it possible to have freely suspended graphene monolayers [40]. For the physics of transport in graphene the two points $\mathrm{K}$ and $\mathrm{K}$ ', named Dirac points, at the corners of the hexagonal Brillouin zone (BZ) are of great importance. 
While in conventional semiconductors charge carriers have a finite effective mass, and their behaviour can be well described by the Schrödinger equation, in graphene the interaction with the honeycomb lattice gives rise to new quasi-particles described at low energy by the Dirac equation, and behaving like relativistic massless particles. The linear energy bands form two symmetric (Dirac) cones that touch at the Dirac points. In this gapless band structure the (Dirac) particles travel with an energyindependent Fermi velocity $v_{F} \approx 10^{6} \mathrm{~cm} / \mathrm{s}$ [41][42][43].

This specific energy dispersion of graphene gives rise to very peculiar transport phenomena as the unusual quantum Hall effect [44], finite conductivity at zero density [45] and Klein tunnelling paradox [46]. The finite conductivity $\sigma_{\min } \sim e^{2} / h$ at the charge neutrality point is a surprising and important result. Several works have investigated the effect of charge inhomogeneity induced by charged impurities and the possible role of electron/hole puddles acting as local pn junctions to explain and quantify accurately this result [47],[48],[49],[50]. It has been shown that the minimum of conductivity close to the Dirac point is dependent on the quality of samples, where electron/hole puddles induced by residual charged impurities or corrugations may hinder the actual value of conductivity at the Dirac point. Beyond these fundamental effects, the excellent transport properties of particles in graphene manifest especially through the very high mobility, not only in suspended graphene sheets [51] but also in graphene layers deposited on a hexagonal $\mathrm{BN}$ substrate where mobility values of $275000 \mathrm{~cm}^{2} \mathrm{~V}^{-1} \mathrm{~s}^{-1}$ and $125000 \mathrm{~cm}^{2} \mathrm{~V}^{-1} \mathrm{~s}^{-1}$ have been achieved at $4.2 \mathrm{~K}$ and room temperature, respectively [52]. The charge transport properties of graphene and graphene-based low-dimensional materials have been reviewed in several articles and textbooks, as e.g. in [53],[54],[55], including the effects of substrate and of different kinds of defect that strongly affect the electron transport properties.

However, in spite of the remarkable advantage of high mobility, graphene has two major drawbacks with a view to thermoelectric applications. First, this material is a gapless semimetal. It is thus difficult to separate the opposite contributions of electrons and holes to the Seebeck coefficient. However, thanks to the strong energy-dependence of the conductivity $\sigma$ near the charge neutrality point [45], a finite value of $S$ can be achieved, consistently with (7). As a result of the balance between this conductivity behavior and the gapless character of graphene, the maximum value of this parameter has been calculated to be smaller than $100 \mu \mathrm{V} / \mathrm{K}$ in pristine graphene [56]. At this point, it is important to remind that the pioneering works of Hicks and Dresselhaus have predicted that even semimetals like Bi may be good thermoelectric materials with high $Z T$ if they are structured in the form of $2 \mathrm{D}$ quantum-well superlattices [57]. Indeed, thanks to the bandgap opening resulting from nanostructuring, these authors showed that $Z T$ can potentially be enhanced to values higher than 5 . Though this optimistic theoretical prediction was done using an oversimplified model and was not quantitatively reproduced either experimentally or by more sophisticated models that consider nonconstant relaxation times and the contribution of high-energy subbands [58], it is legitimate to expect that appropriate nanostructuring may induce high Seebeck coefficient and power factor in graphene, taking advantage of its high mobility. The second major drawback of graphene lies in its very high lattice conductivity. Indeed, it has been shown experimentally that the thermal conductivity of suspended few-layer graphene (FLG) is strongly dependent on the number $n$ of graphene layers. Starting from the graphite value of about $1000 \mathrm{~W} / \mathrm{mK}$ for $n>4$, this parameter reaches a value higher than $4000 \mathrm{~W} / \mathrm{mK}$ for a single layer [59][60], which makes graphene one of the best heat conductor. However, many works have shown that the thermal conductivity of graphene is very sensitive to effects of substrate, strain, defects and isotopes [61][62][63][64]. Actually it is even possible to reduce strongly the thermal conductivity using appropriate nanostructuring and defect engineering [65]. 
Combining all these ingredients regarding the possibility of controlling electrical and thermal transport in graphene nanostructures over a large range, one can expect to achieve high values of $Z T$. With the main goal of recycling energy, the possibility to achieve a high power factor $\sigma S^{2}$ may be also a specific advantage of graphene. Additionally, compared to most of conventional thermoelectric materials, carbon materials would offer the advantage of being abundant, cheap and non-toxic. It is the main aim of this article to review the different approaches that have been investigated experimentally and theoretically to enhance the thermoelectric performance of graphene-based materials.

\section{Thermoelectric properties of monolayer and multilayer graphene}

\subsection{Experiments}

The first measurements of the thermoelectric properties of graphene were published in 2009 [66][67][68]. Actually, these works focussed on the Seebeck and Nernst effects to probe all the components of the thermopower tensor, the diagonal element of which is nothing but the Seebeck coefficient, or simply the thermopower. Indeed, their aim was not to evaluate the potential of graphene for thermoelectric applications but rather to elucidate some details of the electronic band structure and of the ambipolar nature of graphene that cannot be probed by conductance measurements alone. In all these experiments, the structures consisted of a single-layer graphene sheet exfoliated onto a $300 \mathrm{~nm}$ thick $\mathrm{SiO}_{2}$ layer grown on degenerately doped $\mathrm{Si}$ substrate, with a back gate to control the carrier density. All measurements have confirmed that at room temperature the peak value of thermopower reaches about $80 \mu \mathrm{V} / \mathrm{K}$ (See Fig. 1). As expected from this gapless material, the sign of the Seebeck coefficient was found to change as the gate voltage crosses the charge neutrality point (CNP), which indicates the change of sign of the majority charge carrier. Away from the charge neutral region the density-dependence of thermopower behaves as $1 / \sqrt{n}$, where $n$ is the $2 \mathrm{D}$ carrier density, and exhibits a linear temperature-dependence in agreement with the semiclassical Mott formula. As the temperature increases, a deviation from Mott formula was reported [66][67], which is not surprising since this formula, derived mathematically through the Sommerfeld expansion, is valid only at low temperature. This deviation has been well explained theoretically as a consequence of the energy-dependence of electron-impurity scattering [69]. 


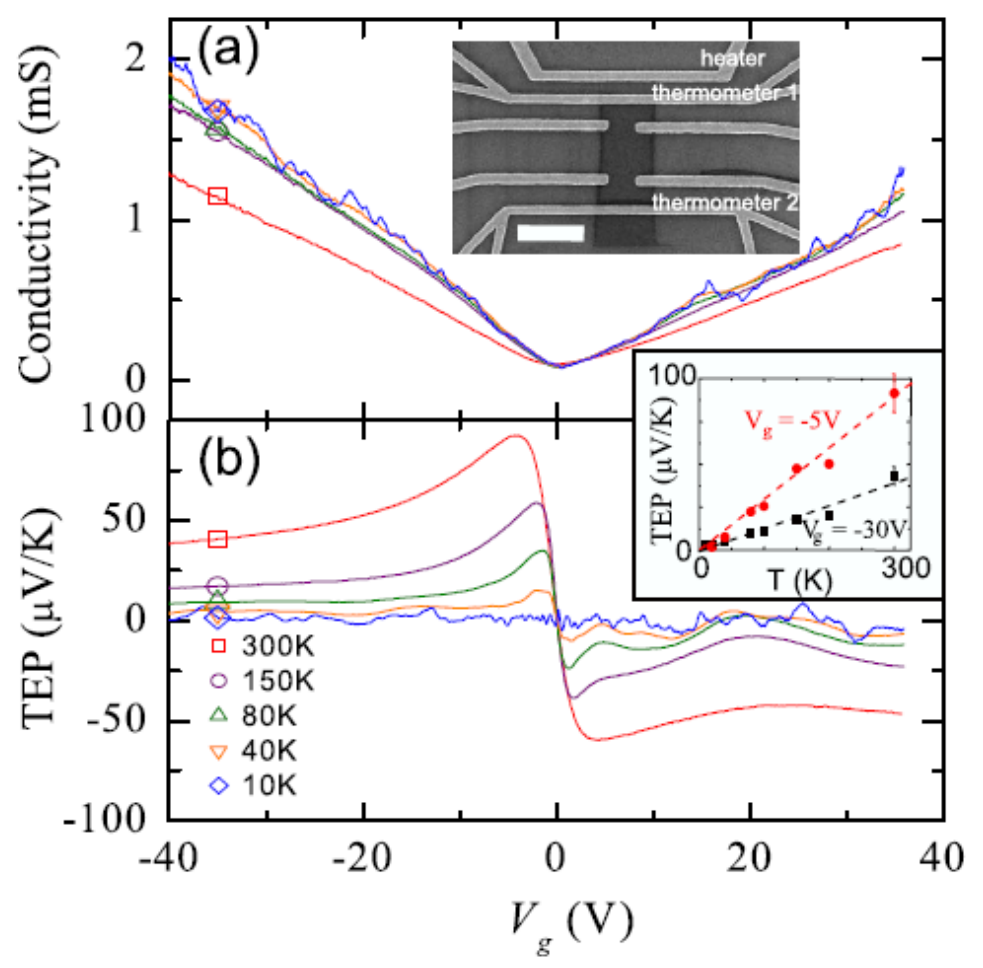

Figure 1. (a) Electrical conductivity and (b) Thermoelectric power (TEP) of a graphene sample as a function of $V_{g}$ for $T=300 \mathrm{~K}$ (square), $150 \mathrm{~K}$ (circle), $80 \mathrm{~K}$ (up triangle), $40 \mathrm{~K}$ (down triangle), and 10 $\mathrm{K}$ (diamond). Upper inset: Scanning electron microscopy image of a typical device, the scale bar is 2 $\mu \mathrm{m}$. Lower inset: Thermopower values taken at $V_{g}=-30 \mathrm{~V}$ (square) and - $5 \mathrm{~V}$ (circle). Dashed lines are linear fits to the data. Reproduced with permission [66]. Copyright 2009, American Physical

Society.

Similar experiments and results were reported in 2010 for massive fermions in bilayer graphene exfoliated onto $\mathrm{SiO}_{2} / \mathrm{Si}$ substrate [70] (see Fig. 2). Again, at low temperature the thermopower follows well the Mott formula. At high carrier density the thermopower shows a linear temperaturedependence, which is consistent with a weak electron-phonon interaction, while at low density a deviation from the Mott formula is observed at high temperature, due to the low Fermi temperature in bilayer graphene. 

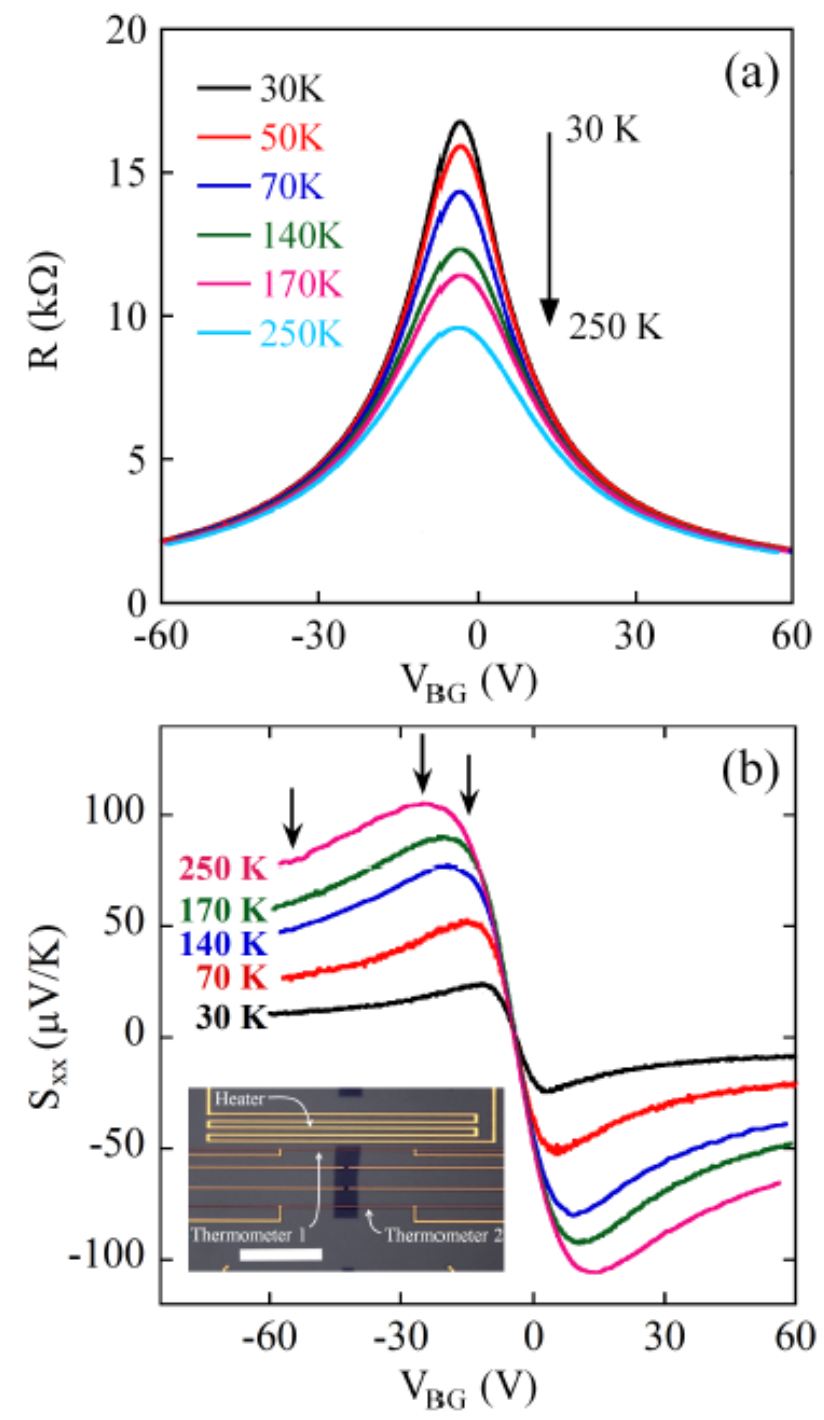

Fig. 2. Thermoelectric measurements in bilayer graphene. (a) Two-terminal resistance $R$ and (b) thermopower as a function of the backgate voltage $V_{B G}$ in zero magnetic field and at various temperatures: $T=30,50,70,140,170$, and $250 \mathrm{~K}$. Inset: optical image of a typical device. Size of the scalebar is $30 \mathrm{~nm}$. Reproduced with permission [70]. Copyright 2010, American Physical Society.

The situation is quite different in high mobility single-layer epitaxial graphene grown on silicon carbide ( $\mathrm{SiC}$ ) substrate [71]. In this case, the temperature dependence of the thermopower shows a strong deviation from the Mott formula. Actually, a quadratic temperature dependence is observed, which is attributed to the screening of charge impurities and its temperature dependence, in agreement with the previous theoretical work of Hwang et al. [69]. This tendency has been confirmed by analysing the thermopower in graphene devices with various degrees of disorder [72]. The Mott relation fails in the vicinity of the Dirac point in high-mobility graphene while it holds in low-mobility graphene where the charged impurities induce relatively high residual carrier density.

The enhancement of thermopower in few-layer graphene films up to $700 \mu \mathrm{V} / \mathrm{K}$ has been obtained after plasma oxygen treatment, which was attributed to the disorder-induced bandgap opening [73]. An increase of thermopower has been also demonstrated in dual-gated bilayer graphene thanks to the bandgap opening resulting from the vertical electric field applied between the two gates [74].

Regarding the differences in thermoelectric effect between monolayer and bilayer graphene, atomicscale mapping of thermopower using scanning tunnelling microscopy (STM) on epitaxial graphene 
has revealed that a big change occurs at the monolayer-bilayer boundaries [75]. Additionally, local thermopower distortions have been attributed to the modification of the electronic structure induced by individual defects as wrinkles of the graphene sheet on the $\mathrm{SiC}$ substrate. It is another way to use thermoelectric effect has a sensitive probe of the quality of graphene layers.

Finally, it is worth mentioning that the thermopower has been found to be very sensitive to the gas molecule charge doping on the surface of graphene grown on $\mathrm{Cu}$ foil by chemical vapour deposition and transferred onto $\mathrm{SiO}_{2} / \mathrm{Si}$ substrate [76]. The observed p-type behaviour under ambient conditions was explained by an electrochemical charge transfer mechanism between graphene and oxygen redox couple, while the n-type behaviour under degassed conditions was ascribed to the electron doping caused by the surface states of the $\mathrm{SiO}_{2} / \mathrm{Si}$ substrate. These effects make this kind of device a potential candidate for gas/chemical sensing.

\subsection{Theory and simulation}

With different levels of approximation in the treatment of scattering, especially due to charged impurities, the first theoretical investigations were very useful to give the main trends of the Seebeck effect in graphene and to discuss the validity of Mott's formula [77][78][79]. An important step toward quantitative results has been taken in 2009 by Hwang et al. who have introduced in their approach of diffusive transport different scattering mechanisms as phonon and charged impurity scattering and their energy-dependence, in particular the dependence induced by screening effects [69]. It allows them to describe correctly the available experimental data, including the possible deviation to the Mott formula, and to make some predictions which were experimentally verified later, as the non-linear temperature dependence of the thermopower and the saturation of thermopower at low density. Quite concomitantly, Yan et al. developed a model based on the self-consistent Born approximation for Dirac fermions including screened charged impurity scattering [80]. They also obtained results of thermopower in excellent agreement with available experimental data even in the low-density regime (See Fig. 3). Then they introduced the influence of a magnetic field in their model to investigate the Nernst effect and transverse thermopower [81]. These works clearly showed that the thermoelectric transport in large graphene samples depends crucially on impurity scattering. Additionally, Zhu et al. have shown that in the presence of magnetic field the thermoelectric coefficients are universal functions of the ratio between the temperature and the disorder-induced broadening of Landau levels [82]. These coefficients exhibit different asymptotic behaviours in different temperature regions and a distinct behaviour in the central landau level has been attributed to an intrinsic property of the Dirac point where both electron and hole Landau levels are degenerate, under the protection of the electronhole symmetry. 


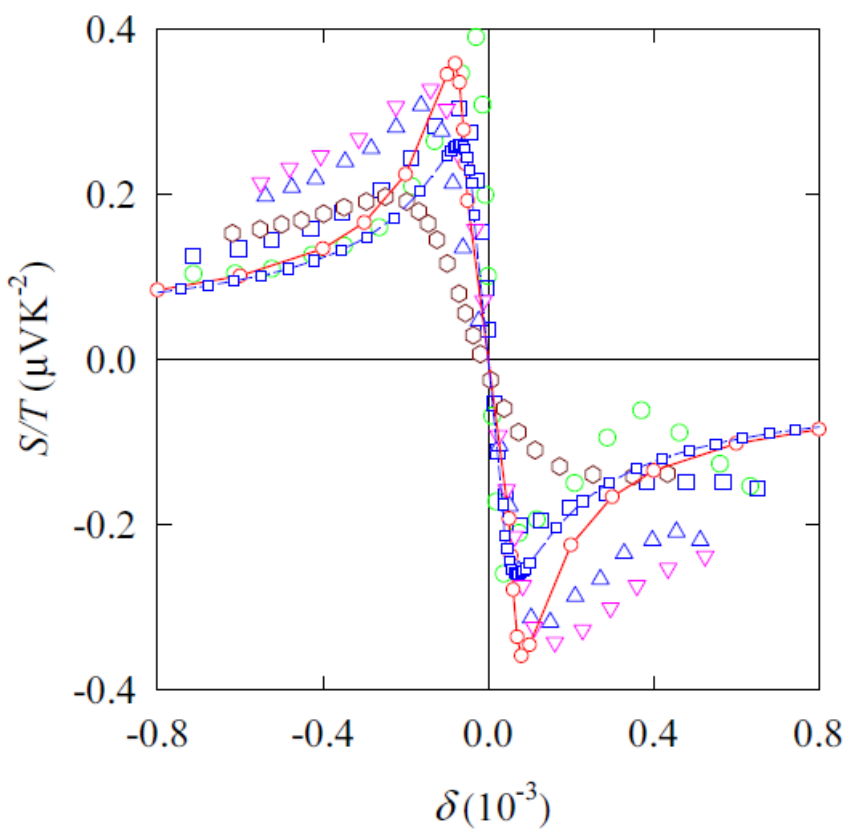

Fig. 3. Linear- $T$ dependence coefficient $S / T$ of thermoelectric power as a function of the electron doping concentration $\delta$. The present calculations (red solid line with circles for renormalized $\mu$ and the blue dashed line with squares for $\mu=E_{F}$ ) are compared with the experimental data in Ref. [66] (circles at $T=150 \mathrm{~K}$ and squares at $T=300 \mathrm{~K}$ ), Ref. [67] (hexagons at $T=255 \mathrm{~K}$ ), and Ref. [68] (up triangles at $T=160 \mathrm{~K}$ and down triangles at $T=280 \mathrm{~K}$ ). Reproduced with permission [80]. Copyright 2009, American Physical Society.

The contribution of the phonon-drag effect that is due to electron-phonon coupling and is known to have an important role in the thermopower of bulk materials [83] and conventional 2D gases [84] has been also evaluated in graphene. By means of balance equation-based simulation, it has been shown that this effect has a significant contribution to the thermopower of graphene only at low temperature $T<10 \mathrm{~K}$ [85], while diffusive processes are dominant at higher temperature. However, it has been calculated recently that in a high-quality graphene sheet with a large phonon mean free path, in the $77 \mathrm{~K}-300 \mathrm{~K}$ range of temperature and at high Fermi level, the contribution to thermopower due to phonon drag may dominate over the diffusive contribution and a sharp increase of the Seebeck coefficient through electron drag by the intrinsic phonons of graphene is expected [86]. This question is still to be clarified and tested experimentally.

The thermopower of bilayer and multilayer graphene has been studied in different works [87][88][89][90]. Under a magnetic field, the disorder-induced broadening of Landau levels has been shown to have in bilayer graphene an effect similar to that in monolayer graphene. The Nernst signal shows a peak at the central Landau level with height of the order of $k_{B} / e$, and changes sign at the other Landau levels, while the thermopower has an opposite behaviour [89] (see Fig. 4). These results are found in good agreement with the experimental ones reported in [70]. If a band gap of $288 \mathrm{meV}$ is induced in a bilayer system by an external vertical field, it has been calculated that the room temperature thermopower is enhanced by a factor of more than 4 compared with that of monolayer graphene and gapless bilayer graphene [87]. A quite systematic study of multilayer graphene has shown that the largest thermopower is actually achieved in biased bilayer graphene [88]. 

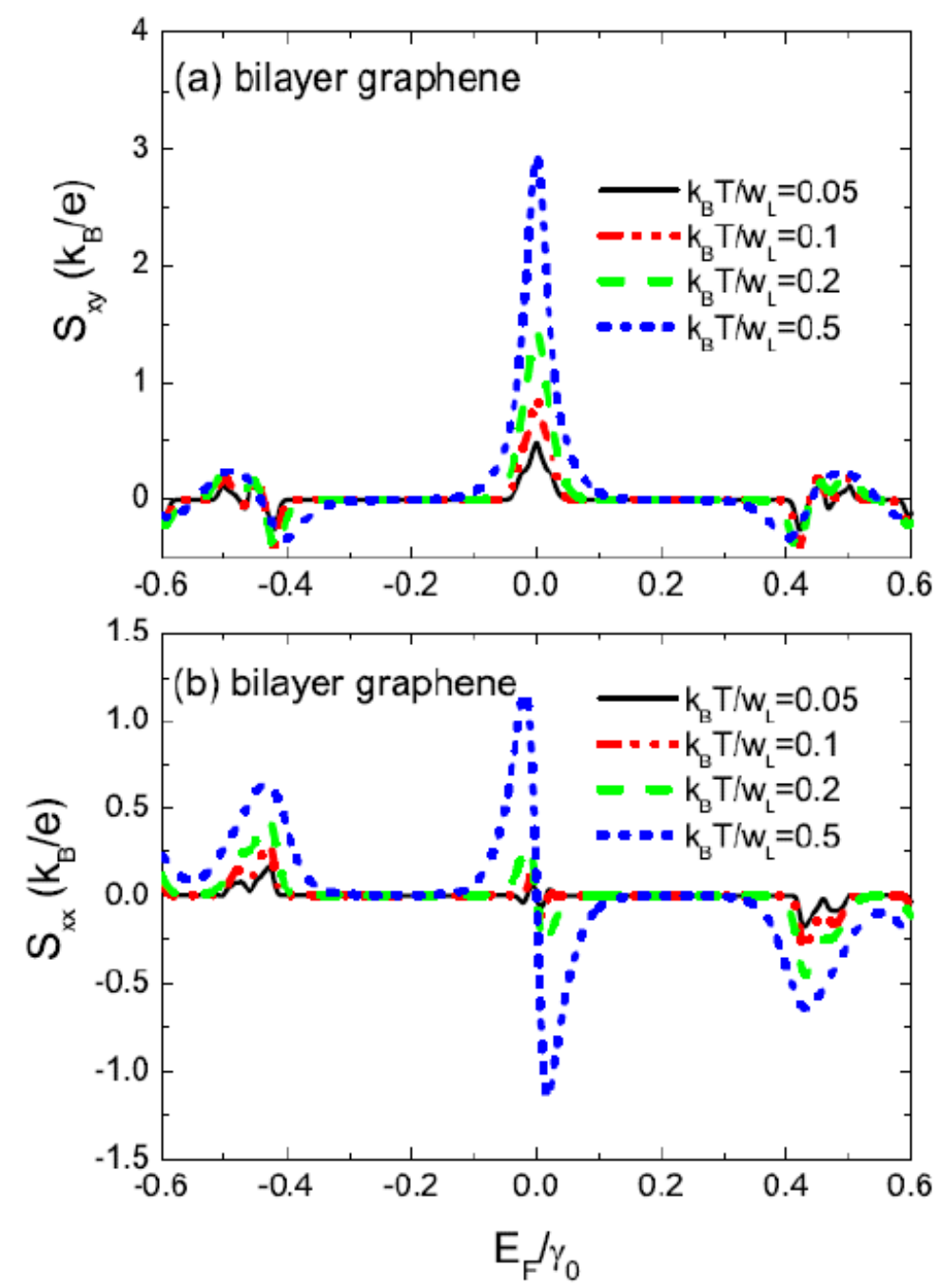

Fig. 4. Thermopower $S_{x x}$ and Nernst signal $S_{x y}$ as functions of the Fermi energy in bilayer graphene and at different temperatures. The system size is taken to be $N=96 \times 48 \times 2$, with magnetic flux $\varphi=2 \pi / 48$, and disorder strength $r_{s}=0.3$ (uniformly distributed positively and negatively charged impurities are considered within this strength) with $W_{L / \gamma_{0}}=0.0376$. Reproduced with permission [89].

Copyright 2011, American Physical Society.

Regarding the effect of bandgap, it is worth mentioning the work of Sharapov et al. based on Kubo calculations showing that in certain conditions, a small bandgap of about $10 \mathrm{meV}$, e.g. due to substrate effect, may be accompanied by the emergence of a new channel of quasiparticle scattering with a relaxation time strongly dependent on energy, which results in the appearance of a high bump in thermopower when chemical potential approaches the gap edge [91]. The authors have suggested to use this Seebeck signal as a sensitive probe of the bandgap in the graphene band structure.

Actually, beyond the interesting physics contained in the thermoelectric coefficients of graphene, the thermoelectric applications of large graphene sheets are very limited due to the gapless nature of this material that makes the thermopower very small compared to that of usual thermoelectric materials. In spite of attempts to generate a bandgap in bilayer graphene using a vertical electric field and thus to enhance the Seebeck coefficient, the high thermal conductivity maintains the thermoelectric figure of merit at much too low values. It is actually possible to reduce the thermal conductivity by substrate engineering [64] but the price to pay is certainly a concomitant reduction of the electrical conductivity [54]. To the best of our knowledge, the effect of substrate on both electron and phonon transport 
properties have been until now studied separately and thus their consequences on the thermoelectric performance of graphene have not been investigated yet. Today, at the present stage of graphene research, it is difficult to predict how the substrate-induced degradation of electrical and thermal conductivities can affect the thermoelectric factor of merit. Strategies to generate a bandgap and to degrade at the same time the thermal conductance have been developed to make graphene a good thermoelectric material. In particular, different approaches of nanostructuring have been investigated, mainly theoretically by means of numerical simulation. We review most of them in the next section.

\section{Nanostructuring graphene to enhance thermoelectric properties}

We now discuss the effect of nanostructuring graphene on the thermoelectric effects and performance. The first sub-section is dedicated to the only Seebeck effect in graphene nanostructures, which involves the charge carrier transport alone. The following sub-sections are dedicated to the theoretical predictions of the full thermoelectric performance of different kinds of nanostructures, including the evaluation of $Z T$ based on the calculation of both electron and phonon transport.

As an introduction to this section, we would like to summarize briefly the main numerical methods used to calculate the electron and phonon transport properties in graphene nanostructures.

Different semi-classical and quantum approaches have been developed to describe the phonon transport in nanostructures such as the Monte Carlo solution of the Boltzmann equation [92], the molecular dynamics [93] [94], the transfer matrix [95], the order-N real-space Kubo formalism [96], or the non-equilibrium Green's function (NEGF) method to solve either a semi-empirical [97][98] or a first-principles [99] force constant matrix.

The NEGF method is also widely used to simulate the quantum transport of charges in graphene devices by considering either the continuous Dirac Hamiltonian in large graphene samples [100][101][102] or an atomistic tight-binding Hamiltonian in GNRs and other nanostructures [103][104][105][106][107]. It has thus been used also to compute simultaneously electron and phonon transport in graphene structures, which gives access to their thermoelectric properties [108][109][110]. Actually, a majority of the works presented below are based on this method.

In most of them, a simple nearest-neighbour tight-binding (NNTB) Hamiltonian without edge relaxation was used for electrons, while a force constant model (FCM) including the fourth nearestneighbours was considered for phonons [108][110]. It should be noted that a third NNTB model was sometimes used for electrons [110], while a fifth nearest-neighbours FCM has been used for phonons [98][109][111]. Today, in small structures, the NEGF method for transport is frequently coupled with first-principles description of the materials [99].

Given the large electron and phonon mean-free paths of electrons and phonons in graphene [112][113], the simulation of transport is usually done in the ballistic approximation. Basically, the electron (phonon) transmission function $T_{e}\left(T_{p}\right)$ is deduced from the Green's functions of the electron Schrödinger equation (lattice dynamic equation). The Landauer formalism is then applied to calculate the phonon and electron fluxes and all related quantities. The electronic conductance $G$ is then deduced as a function of the Fermi energy $\mu$ as

$$
G(\mu)=\frac{2 e}{h} T_{e}(\mu) .
$$

The Seebeck coefficiant $S$ and the electronic contribution to the thermal conductance $K_{e}$ can be derived via the intermediate $L_{n}$ functions defined as [114][115] 


$$
L_{n}(\mu, T)=\frac{2}{h} \int T_{e}(E)(E-\mu)^{n} \frac{-\partial f(E, \mu, T)}{\partial E} d E,
$$

where $f(E, \mu, T)$ is the Fermi-Dirac distribution function at temperature $T$. Finally, $S$ and $K_{e}$ are expressed and computed as

$$
S(\mu, T)=\frac{1}{e T} \frac{L_{1}(\mu, T)}{L_{0}(\mu, T)},
$$

and

$$
K_{e}(\mu, T)=\frac{1}{T}\left[L_{2}(\mu, T)-\frac{L_{1}(\mu, T)^{2}}{L_{0}(\mu, T)}\right] .
$$

The phonon contribution to the thermal conductance may be expressed in a Landauer form as

$$
K_{p}=\int T_{p}(\omega)\left[\frac{\partial n(\omega, T)}{\partial T}\right] \frac{\hbar \omega}{2 \pi} d \omega,
$$

where $n(\omega, T)$ is the Bose-Einstein distribution function. This general expression can be conveniently rewritten in the form [116]

$$
K_{p}(T)=\frac{1}{8 \pi k_{B} T^{2}} \int_{0}^{\infty} \hbar^{2} \omega^{2} \frac{T_{p}(\omega)}{\sinh ^{2}\left(\frac{\hbar \omega}{2 k_{B} T}\right)} d \omega
$$

Finally, by regrouping (8), (10), (11) and (13), the thermoelectric figure of merit $Z T$ (2) can be computed.

\subsection{Seebeck effect}

In 2007, Dragoman and Dragoman suggested that it was possible to hugely enhance the thermopower in a nanostructure consisting of metallic electrodes periodically patterned over a graphene sheet [117]. By using a transfer matrix calculation, they showed that the ballistic transmission of this superlattice can be engineered into a transmission gap surrounded by a series of delta-like peaks caused by the interference of electron waves, similar to the case of a multi-layered Bragg reflector in optics. A simple estimation of the thermopower from a Mott-like formula then leads to peak values about $30 \mathrm{mV} / \mathrm{K}$. Using a similar approach, it has been predicted that by introducing a defect barrier in such a graphene superlattice, the maximum value of the thermopower may reach $260 \mathrm{mV} / \mathrm{K}$ at room temperature [118]. However, in spite of these extremely high values of thermopower, since the phonon transport is certainly weakly affected, this kind of nanostructuring can probably not be a good candidate for thermoelectric energy conversion that requires high $Z T$.

By means of the non-equilibrium Green's function method to solve a nearest-neighbour tight-binding Hamiltonian, Xing et al. have calculated the thermoelectric (Nernst and Seebeck) coefficients in quite large $(W \approx 15$ to $34 \mathrm{~nm}$ ) ballistic graphene nanoribbons (GNRs) [119]. In zero magnetic field, the Seebeck effect was shown to be strongly dependent on the chirality of the ribbon. For the zigzag edge ribbons, the peaks of Seebeck coefficient are equidistant while they are irregularly distributed for armchair edge ribbons. Interestingly, in semiconducting armchair GNRs, the Seebeck coefficient can be quite large near the Dirac point due to the energy gap. When increasing the magnetic field the behaviour of the thermopower becomes independent of the chirality. For large and nearly gapless 
GNRs, Divari and Kliros used the linear response theory to examine the validity of Mott's relation [120]. As expected, they observed that the Mott's formula gives good results at low temperature only. As the temperature increases, a nonlinear temperature dependence of thermopower appears and is more pronounced at low electron density. It should be noted that in short GNRs, Fabry-Perot oscillations are visible at low temperature in the evolution of the thermopower as a function of chemical potential. The period of oscillations are well predicted by geometrical considerations of propagating modes.

The case of narrow and long armchair GNRs has been studied by Nissimagoudar and Sankeshwar by including in their calculation the effects of scattering by edge roughness, impurities and both acoustic and optical phonons [121]. They found again that Mott's formula overestimates the thermopower for temperatures $T>75 \mathrm{~K}$. At room temperature, the thermopower reaches $42 \mu \mathrm{V} / \mathrm{K}$ for a width $W=5 \mu \mathrm{m}$ and $60 \mu \mathrm{V} / \mathrm{K}$ for $W=3 \mathrm{~nm}$. However, it has been shown that for short and narrow GNRs in ballistic regime, much higher values of thermopower may be achieved, with a maximum value that is a linear function of the energy gap [98]. For instance, a maximum value of $1.2 \mathrm{mV} / \mathrm{K}$ for a bandgap of $350 \mathrm{meV}(W=1.84 \mathrm{~nm})$ has been predicted.

Though other works have shown that very high values of thermopower $(\approx 1 \mathrm{mV} / \mathrm{K})$ may be achieved in narrow GNRs and other graphene nanostructures, to make these nanomaterials suitable for thermoelectric energy conversion with high enough $Z T$, it is necessary to reduce concomitantly the thermal conductance without degrading strongly the electronic conductance. Many works have been dedicated to take up this challenge. The rest of this section is an overview of this research area.

\subsection{Nanoribbons}

The first attempt to investigate the full thermoelectric properties of short ballistic GNRs was reported by Ouyang and Guo in 2009 [56]. Actually, this work did not suggest that nanostructuring graphene into narrow ribbons may improve significantly the thermoelectric performance of graphene in spite of the enhancement in thermopower. It even showed that edge roughness and lattice vacancies increase the thermopower but decrease the thermoelectric figure of merit $Z T$ because the decrease in the electronic conductance outweighs the decrease in the thermal conductance and the increase in the thermopower. However, this work and the approach used has inspired many further investigations which finally showed that a large $Z T$ enhancement is possible using graphene nanostructures. Zigzag GNRs (ZGNRs) do not exhibit any significant thermoelectric figure of merit because they are gapless. In narrow armchair GNRs (AGNRs) with finite bandgap, $Z T$ is typically of the order of only 0.1 at room temperature [98]. Higher $Z T$ values were predicted in large ribbons [122] but the phonon contribution to the thermal conductance was not included in this calculation. In some cases, uniaxial tensile strain applied along the main axis of AGNRs may enhance the figure of merit, which is due either to an increase in the electronic transmission around the conduction and valence band edges, or to an increase in the bandgap that reduces the unfavourable bipolar transport [123].

To enhance further the figure of merit $Z T$, it is necessary to design more sophisticated narrow ribbons likely to degrade strongly the phonon contribution to the thermal conductance while retaining high electron conduction properties. Several works have suggested to use multi-junction GNRs with alternate sections of different width, different chirality, or different chirality and width [124][98][109][125][126]. Let's consider the case of a mixed GNR (MGNR) consisting of a superlattice of alternate sections of 8-AGNR and 20-ZGNR, where $n=8$ and $m=20$ are the number of dimer atoms in the unit cell defining the width of the ribbons, as schematized in Fig. 5(a). The behaviour of this MGNR is compared with that of a perfect 8-AGNR in Fig. 5(bcd), for a device 
length, i.e. the distance between two leads, smaller than $100 \mathrm{~nm}$. First, the phonon thermal conductance $K_{p}$ in MGNR is drastically reduced compared to that of AGNR, which is a consequence of the mismatch of phonon modes in the different sections of MGNR. Second the thermopower is not only significantly increased in MGNR but it presents strong oscillations between negative and positive values from $-0.3 \mathrm{mV} / \mathrm{k}$ to $0.3 \mathrm{mV} / \mathrm{K}$ [98], which is a typical behaviour of a multi-barrier system in resonant tunnelling regime [127]. This resonant effect appears also on Fig. 5(c) where the electronic conductance of MGNR, though reduced compared to that of AGNR, exhibits high peak values that correspond to resonant transport within the minibands of the superlattice. As illustrated on Fig. 5(a) in the map of the local density of states at energy $E=0.05 \mathrm{eV}$ (in the first conductance peak), the resonant tunnelling takes place between states localized at the edges of the zigzag sections. However, the resonant states may be localized differently at other energies [98]. The combined effect of the thermopower enhancement and reduced thermal conductance finally boosts $Z T$ that exceeds unity for the MGNR structure at room temperature, as shown in Fig. 5(d). These results were obtained from a fifth nearest-neighbour force-constant model and a nearest-neighbour tight-binding electron Hamiltonian including armchair edge relaxation, both solved within the NEGF formalism [98][109][128].

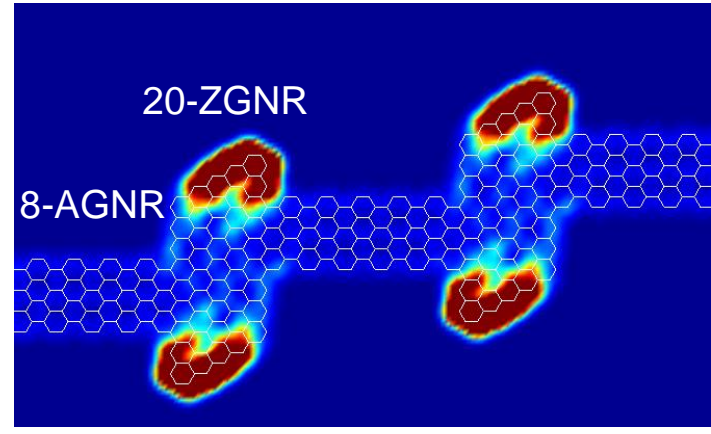

(a)

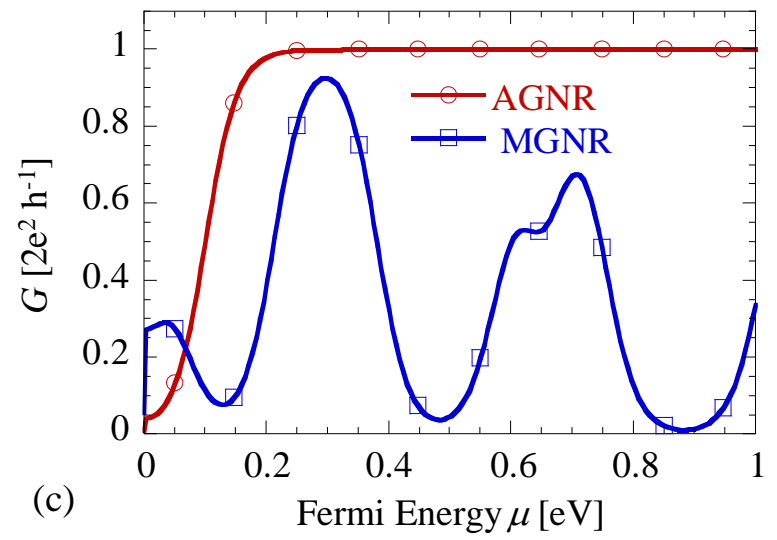

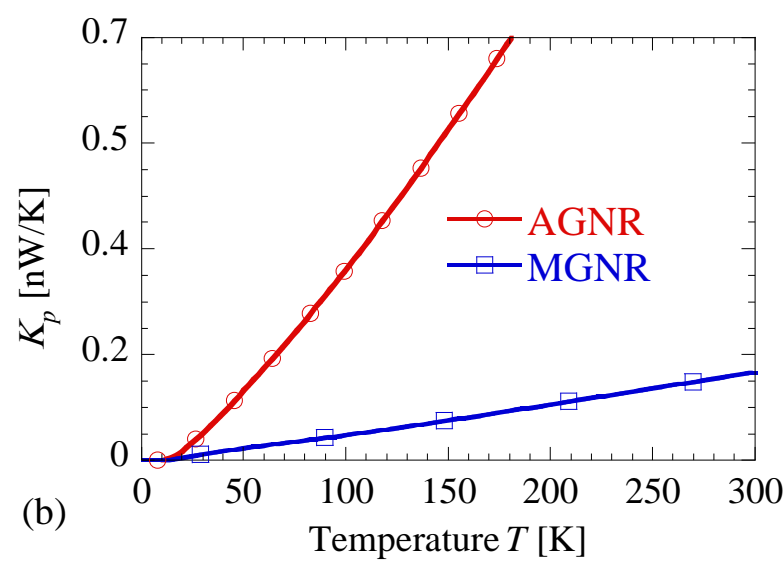

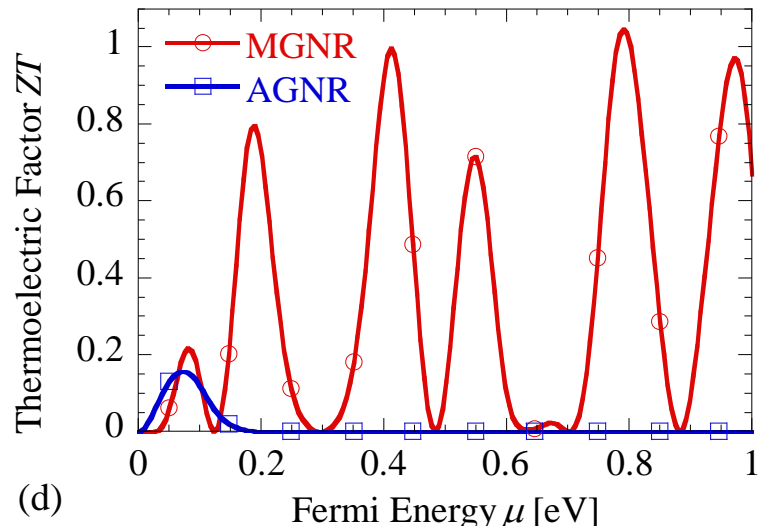

Fig. 5. (a) MGNR consisting of alternating 8-AGNR and 20-ZGNR sections, and local density of states at energy $E=0.05 \mathrm{eV}$. Red and blue areas correspond to high and low DOS, respectively. (b) Phonon contribution to the thermal conductance as a function of temperature in MGNR and 8-AGNR.

(c) Electronic conductance and (d) figure of merit $Z T$ as a function of chemical potential in both GNRs at room temperature. Further details in Ref. [98].

Very promising results were also obtained in kinked (or chevron-type) GNRs or graphene nanowiggles (GNWs). This shape of ribbons was suggested by the bottom-up chemical synthesis of 
such ribbons with atomic precision reported by Cai et al. [129]. This method has been recently further improved, which makes it possible to envision the fabrication of perfectly controlled graphene nanostructures with sophisticated design [130]. The first investigation of thermoelectric properties of this type of ribbon showed a significant reduction of thermal conductance compared to its straight counterpart and the value $Z T=0.6$ was obtained at room temperature [124]. Additionally, it has been shown that the thermoelectric properties of kinked GNRs is less sensitive to the edge chirality than that of straight GNRs [131]. By combining DFT with NEGF formalism, Liang et al. investigated several kink configurations and demonstrated that the phonon contribution to the thermal conductance may be dramatically degraded while the electronic conduction is preserved [132]. In particular, the resonant tunnelling effect between alternate parallel and oblique sectors contributes to maintaining good electronic transport properties, as already demonstrated in other types of ribbons consisting of alternate zigzag and armchair sections [98]. Liang et al. obtained a maximum $Z T=0.79$ at room temperature and demonstrated that the value $Z T=1$ may be reached if the kinked-GNR undergoes a structural dislocation that further reduces the thermal conductance by a factor of about 2 [133]. Recently, Sevinçli et al. have shown that by combining this chevron-type geometry with ${ }^{14} \mathrm{C}$ and ${ }^{12} \mathrm{C}$ isotope cluster engineering (Fig. 6), it is possible to further increase the figure of merit $Z T$ [134]. A detailed investigation of the length dependence at different temperatures led to the conclusion that $Z T \geq 2$ is achievable at room temperature in the range of length $100 \mathrm{~nm}-1 \mu \mathrm{m}$ and $Z T \geq 2$ was predicted for $T=800 \mathrm{~K}$ and $L \simeq 75 \mathrm{~nm}$ (Fig. 7).

a
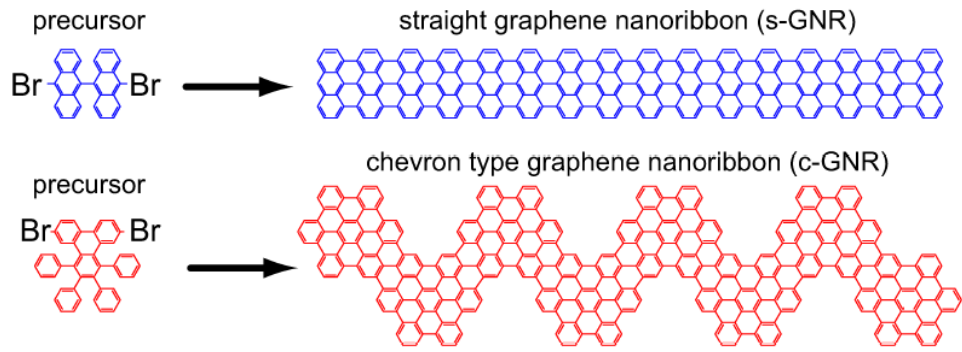

b
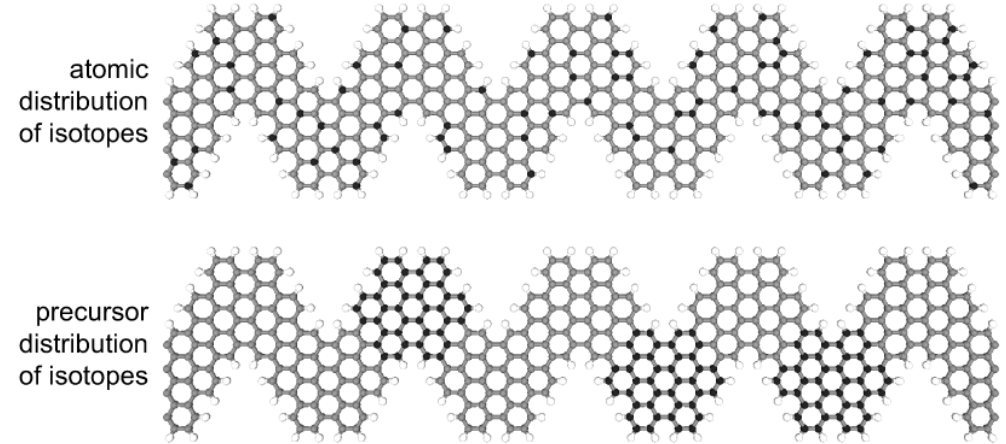

Fig. 6. Structural aspects and isotope distribution types of bottom-up fabricated graphene nanoribbons

(GNRs). (a) Structures of precursors and corresponding straight and chevron type graphene nanoribbons, s-GNR and c-GNR. (b) Heavy isotopes can be distributed at the atomic or precursor level. Grey, 12C; black, 14C; white H. Reproduced with permission [134]. Copyright 2013, Nature Publishing Group. 


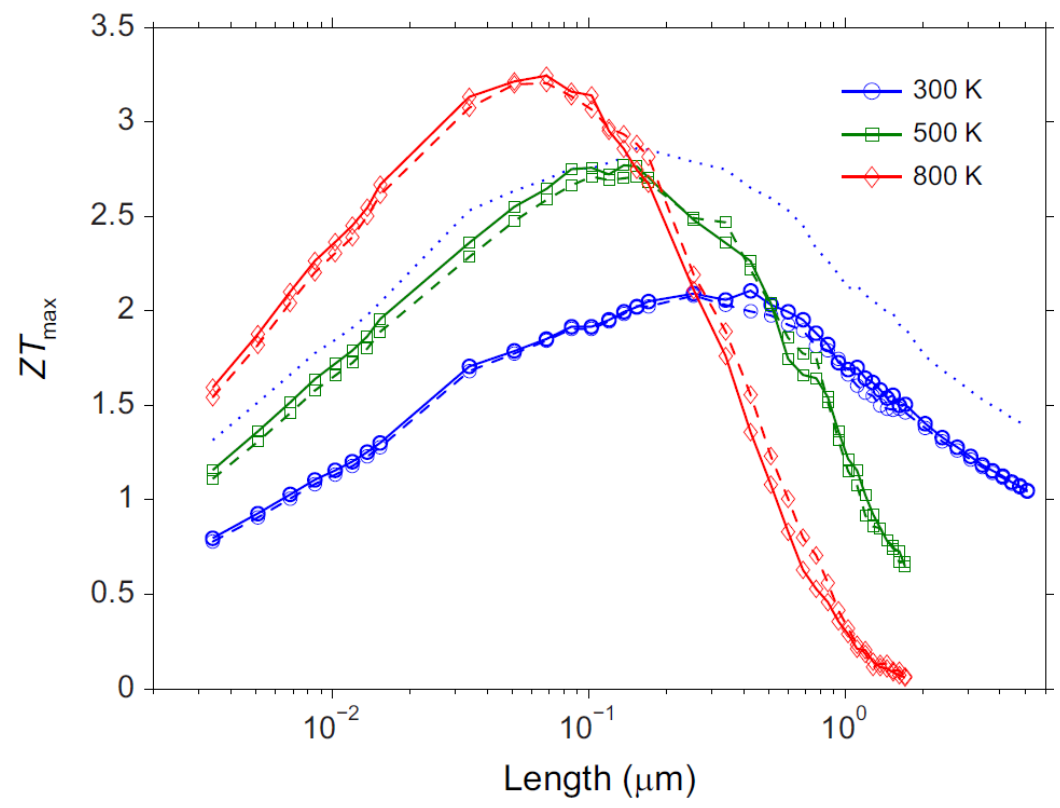

Fig. 7. Maximum $Z T$ as a function of length at different temperatures in structures schematized in

Fig. 6. Maximum $Z T$ achievable when Anderson type disorder is introduced in the electronic

Hamiltonian. The variation of onsite energies are set equal to the temperature, $\sigma=k_{B} T$. Solid lines represent hole-like transport while the dashed lines are for electron-like particles. The $Z T_{\max }$ values shown are realized inside the band gap, i.e $|\mu|<0.75 \mathrm{eV}$ with respect to the mid-gap, except for the dotted curve $(T=300 \mathrm{~K})$ when $|\mu|>1$. Reproduced with permission [134]. Copyright 2013, Nature Publishing Group.

Different suggestions of defect engineering were also investigated to enhance the thermoelectric properties of GNRs [108][109][135]. Sevinçli and Cuniberti have shown that in edge-disordered ZGNRs with a length greater than a few hundred nanometers, the phonon thermal conductance can be strongly suppressed while electronic transport stays intact within the first conduction plateau. They predicted that thanks to a sharp peak in the transmission spectrum, a high value $Z T=4$ can be achieved at room temperature in 20-ZGNRs of length $4 \mu \mathrm{m}$, but at energies higher than $0.5 \mathrm{eV}$, i.e. difficult to reach [108]. By considering the presence of extended line defects, Karamitaheri et al. demonstrated that the value $Z T \geq 2$ is achievable at low energy in ZGNRs of $2 \mu$ m-length [135]. However, it should be mentioned that in both cases, the calculation was made by neglecting the effect of electron-phonon scattering though it may be significant in such long GNRs.

\subsection{Single molecule and quantum dot nanojunctions}

Ultra-scale structures were also suggested by Nikolić et al. to optimize the thermoelectric performance, and investigated by means of NEGF-DFT simulation [136][99]. They consist of two semi-infinite metallic ZGNR leads attached to a single organic molecule that is either a 18-annulene (a ring of 18 carbon atoms) or a 10-chain (a chain of 10 carbon atoms) (see Fig. 8). It has been shown in particular that, first, the highly transparent GNR-molecule contact allows for evanescent wavefunctions to penetrate from the ZGNR leads into the HOMO-LUMO gap of the small molecule, which generates a transmission resonance known as a favourable ingredient to optimize the thermopower. Second, the mismatch in vibrational properties of the semi-infinite ZGNR lead and the molecule contributes to reduce the phonon thermal conductance across the junction by a factor of about three when compared to infinite ZGNRs. It results in figures of merit $Z T \simeq 0.4$ at room temperature and $Z T \simeq 3$ at $T=10 \mathrm{~K}$. 

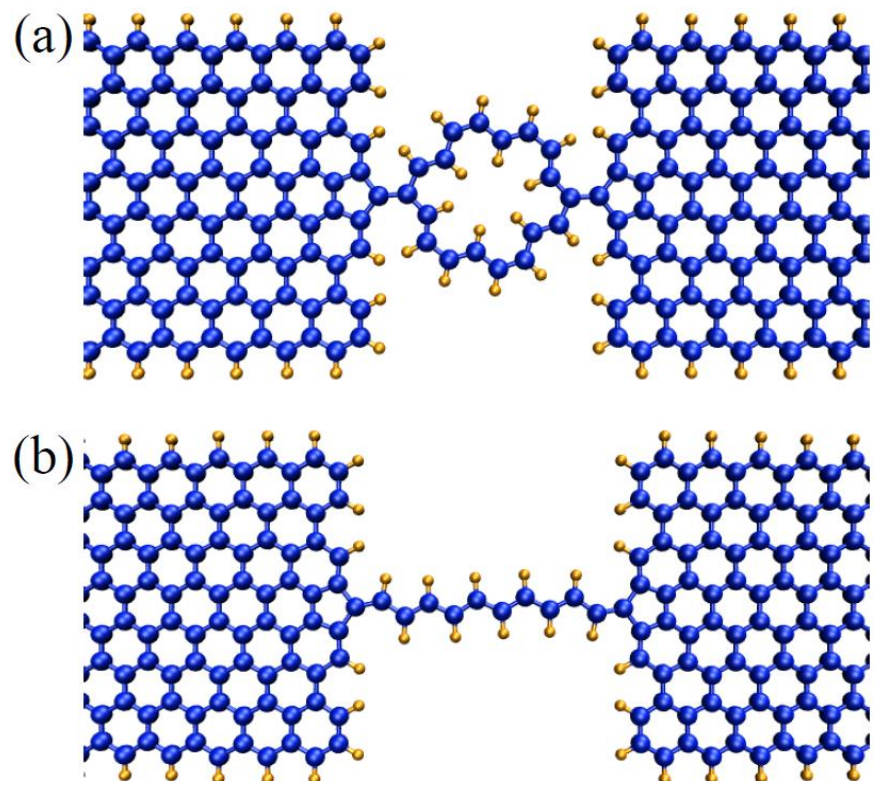

Fig. 8. Schematic view of (a) ZGNR|18-annulene|ZGNR and (b) ZGNR|C10|ZGNR single-molecule nanojunctions. The contact between the source and the drain 8-ZGNR (consisting of eight zigzag chains) metallic electrodes and molecules is made via five-membered rings of carbon atoms (dark blue). The hydrogen atoms (light yellow) are included to passivate the edge carbon atoms in the nanoribbon or dangling bonds in the molecules. The distance between ZGNR electrodes is $d=1.29 \mathrm{~nm}$ for the device in panel (a) and $d=1.59 \mathrm{~nm}$ for the device in panel (b). Reproduced with permission [99]. Copyright 2012, Springer.

In the same spirit, thermoelectric performance of graphene quantum dots consisting of GNRs with narrow constrictions has been investigated using standard TB-FCM-NEGF simulation [137]. The authors have shown that, when decreasing the width of the constrictions, the thermal conductance of a GNRs can be strongly reduced while the power factor remains high as compared with that obtained for the pristine nanoribbon counterpart. As a result, the thermoelectric figure of merit can be enhanced to $Z T \simeq 0.8$ at room temperature.

\subsection{Nanomeshes (anti-dot lattices)}

It is worth mentioning the idea to use GNRs containing a periodic array of vacancies in the centre [109] or perforated by an array of nanopores (or anti-dots) in the interior [138][139]. In the latter case, it was shown that due to the fact that local charge current density is peaked around their edges, nanopores do not impede such "edge currents" while drastically reducing the phonon conduction in sufficiently long zigzag or chiral GNRs [139]. The authors arrived to the conclusion that if the pore diameter takes a random value within some interval and the distance between the pores is varied, it is possible to achieve a very high figure of merit $Z T=5$ at both $T=77 \mathrm{~K}$ and $300 \mathrm{~K}$ for a ZGNR of $1 \mu \mathrm{m}$-length.

Recently, Chang et al. studied the behaviour of $6 \mathrm{~nm}$-wide ZGNRs with nanopores and heavy adatoms [140]. They have shown that the adatoms locally enhance spin-orbit coupling in graphene, which converts it into a two-dimensional topological insulator with a band gap in the bulk and robust helical edge states. These edge states have the property to carry electrical current and generate a high power factor per helical conducting channel. Additionally, the array of nanopores suppresses the lattice thermal conduction through the centre of the ribbon. Numerical simulations combining DFT and 
NEGF methods demonstrated a thermoelectric figure of merit reaching its maximum $Z T \simeq 3$ at low temperature $T=36 \mathrm{~K}$.

The idea to punch a periodic array of nanopores can be of course extended to $2 \mathrm{D}$ graphene sheets to form a graphene nanomesh (GNM), which offers a promising way of bandgap nano-engineering suitable for large graphene areas (see Fig. 9). This type of nanostructuring has been demonstrated experimentally by different methods [141][142][143][144] and investigated theoretically [145][146][147][148][149]. It opens new routes of band structure engineering for graphene applications [150], including tunnel diodes with high peak-to-valley ratio [107], electron waveguides [151], plasmonics in microwave and $\mathrm{THz}$ regions [152] or transistors with high on-off ratio and maximum oscillation frequency $f_{\max }$ [153]. It offers also some opportunities for thermoelectric devices designed on large graphene sheets likely to deliver higher output power than GNR devices, which has been investigated theoretically by some groups [110][154].

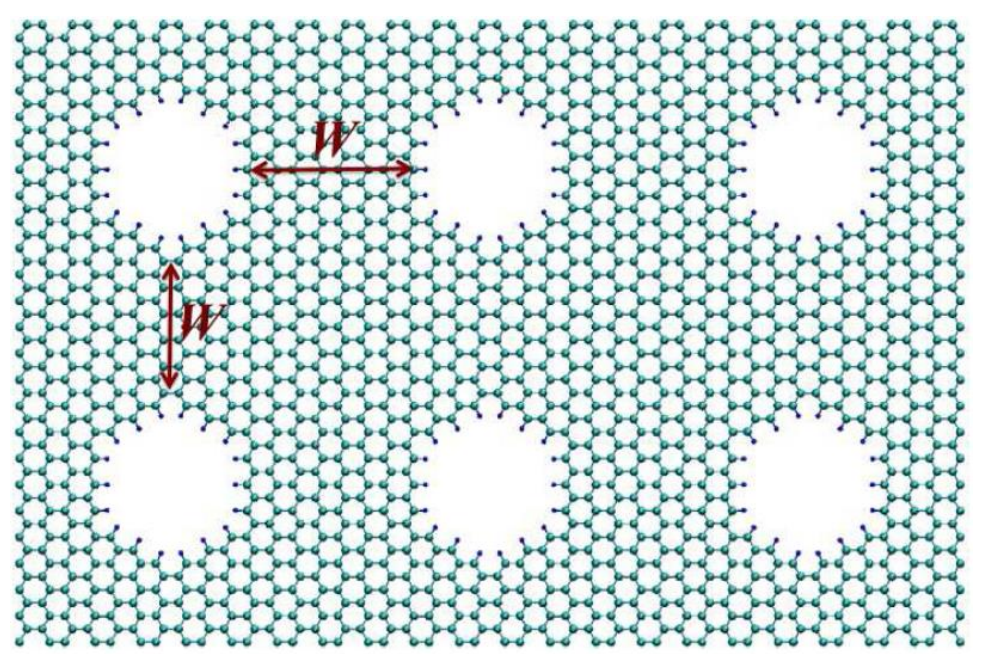

Fig. 9. A typical H-passivated GNM system. Here the big cyan (small blue) spheres represent $\mathrm{C}(\mathrm{H})$ atoms, and $\mathrm{W}$ denotes the neck width along the zigzag and armchair directions. Reproduced with permission [147]. Copyright 2012, American Physical Society.

Gunst et al. have shown that both electronic and thermal transport properties converge rapidly toward the bulk limit with increasing the length of the lattice [154]. Only six repetitions of the fundamental unit cell are required to recover the electronic band gap of the infinite lattice as a transport gap for the finite lattice.

Further improvements may be expected by considering hydrogen passivation of dangling bonds likely to suppress the thermal conductance [110] or by including isotope scattering and anharmonic interactions [154].

\subsection{Vertical junctions in multilayer graphene}

The vertical transport across bilayer or multilayer graphene systems has been recently investigated in terms of thermoelectric effects [155]. In the case of junctions consisting of two partially overlapped graphene sheets, it was shown that because of the weak van-der Waals interactions between graphene layers, the phonon conductance in these junctions is strongly reduced compared to that of a single graphene layer, while the electrical conduction is weakly affected. Depending on the length of the overlap region, the phonon conductance can be reduced by about $92 \%$ at room temperature. Since a finite energy gap is required to get a significant thermopower and figure of merit $Z T$, the concept has 
been applied to 1D 12-AGNRs. The resulting vertical junction exhibits a Seebeck coefficient $S$ as high as $0.9 \mathrm{MV} / \mathrm{K}$ and a maximum figure of merit $Z T=1$ and $Z T=1.7$ at $T=300 \mathrm{~K}$ and $T=600 \mathrm{~K}$, respectively. Finally, this idea has been applied to junctions of 2D GNM lattices. A square GNM lattice has been considered with periodic nanoholes obtained by the removal of 26 carbon atoms in a super-cell of 140 atoms. The phonon thermal conductance is reduced by a factor greater than 10 compared to a single GNM lattice structure, while the maximum thermopower reaches $1 \mathrm{mV} / \mathrm{K}$. It results in a maximum figure of merit $Z T>1$ and $Z T=3.2$ at $T=300 \mathrm{~K}$ and $T=600 \mathrm{~K}$, respectively, which are probably the highest values reported for a $2 \mathrm{D}$ graphene device.

\subsection{Spin caloritronics in graphene}

Another promising field of research in thermoelectrics has recently emerged under the terminology of spin caloritronics, combining both spintronics and thermoelectrics to control thermally-induced spinpolarized currents. The relevant effects in this field are classified into "independent-electron effects", as the spin-dependent Seebeck effect, if two parallel spin-transport channels are formed with different thermoelectric properties, and "collective effects", as the spin Seebeck effect, caused by spin waves. The latter effect, that converts a heat current into a spin current, occurs in a paramagnetic contact to a ferromagnet with a temperature bias. It is described in terms of a spin current injected into the normal metal by the ferromagnet that is transformed into an electric voltage by the inverse spin Hall effect [156][157]. It has been first demonstrated in permalloy [158] and later in the magnetic insulator $\mathrm{LaY}_{2} \mathrm{Fe}_{5} \mathrm{O}_{12}$ [159] and in the ferromagnetic semiconductor GaMnAs [160]. Recently, the opportunity to easily tune the electronic properties of graphene have been used to investigate theoretically the spin caloritronics properties of this material.

Basic studies were first focused on the spin-polarized transport through a magnetic zigzag GNR under temperature bias [161][162][163]. First-principles calculations have shown that the spin Seebeck effect in this type of GNR can be attributed to the asymmetric electron-hole transmission spectra of spin-up and spin-down electrons, and can be modulated by tuning the gate voltage [161]. Additionally, in terms of thermal magnetoresistance, high performance has been predicted in ZGNR heterojunction with appropriate design of Hydrogen passivation [164] and in ZGNR with Mn adatoms at single vacancies [165].

Similar effects have been studied also in $2 \mathrm{D}$ normal/ferromagnetic/normal graphene (NG/FG/NG) structures, where the local ferromagnetism is assumed to be resulting from the proximity effect induced by a magnetic insulator while a finite bandgap is supposed to be generated by substrate effect [Niu_2013]. It was predicted that (i) the thermally-induced spin-up and spin-down currents flow in opposite directions, which results in a pure spin current, (ii) the spin-polarized current can be tuned by the gate voltage and (iii) a large thermal magnetoresistance can be achieved in a NG/FG/NG/FG/NG structure.

More detailed investigations including the evaluation of the thermoelectric figure of merit were recently reported in ZGNR-based spin valves [166] and in ZGNRs with antidots (nanopores) [167]. In the latter case, it has been shown that high values of charge $Z T_{c}$ and spin $Z T_{s}$ figures of merit can be achieved, i.e. higher than 0.6 at room temperature and even much higher $(>5)$ at low temperature $T=100 \mathrm{~K}$.

Actually, further theoretical investigations and experimental demonstrations are still needed to assess the relevance of the concept of spin caloritronics in graphene. 


\section{Beyond graphene: thermoelectrics in other 2D materials}

Since the discovery of graphene and of the "wonderful world" of 2D layered materials, plenty of new 2D materials were proposed and unveiled, such as graphene oxide, hybrid hydrogenated graphene (in the form of graphane or graphone), hexagonal boron nitride monolayers, hybrid graphene/boron nitride monolayers, thallium nitride monolayers, aluminum nitride monolayers, germanium carbide monolayers, gallium nitride monolayers, silicene, phosphorene, molybdenum disulfide, and other transition metal dichalcogenides. New forms of non-natural carbon allotropes related to graphite/graphene, graphyne and graphdiyne have also been the subjects of interest due to their unique structures and intriguing properties. Here, we review some of the most promising of them in terms of thermoelectric properties.

\subsection{Hybrid graphene structures and other layered carbon allotropes}

The properties of graphene can be tuned via various chemical functionalization methods. In particular, graphene can react with atomic hydrogen, which transforms this highly conductive gapless semimetal into a semiconductor or an insulator [168][169]. There are two typical forms of graphene hydrogenation. One form leads to graphane (GA), a fully double-side hydrogenated graphene sheet with hydrogen atoms bonded to carbon atoms on both sides alternatively, where all the $\mathrm{sp}^{2}$ bonds are transformed into $\mathrm{sp}^{3}$ ones [168][170]. The second one, called graphone (GO), is a single-side hydrogenated graphene sheet, obtained by utilizing the reversibility of the hydrogenation process, i.e., by removing all the hydrogen atoms at one side of GA, in which half of $\mathrm{sp}^{3}$ bonds are transformed back into $\mathrm{sp}^{2}$ bonds [171][172]. It may result in the change of the magnetic property from antiferromagnetic to ferromagnetic [171]. Since hydrogenation is reversible and controllable [173][174], it may be used to tune the bandgap of the material and its electronic properties on a wide range.

By using molecular dynamic simulation, it has been shown that, depending on the hydrogenation form and coverage fraction, periodic ripples and buckling may appear and the thermal conductivity of hydrogenated graphene sheets can be $30 \%$ to $75 \%$ less than that of the pure graphene sheets [175]. From DFT-NEGF simulation, Ni et al. have found that the thermoelectric properties of armchair graphane nanoribbons (AGANRs) are higher than that of AGNRs and further enhanced in the presence of disorder [176]. By introducing disorder in the form of imperfect saturation of the hydrogen-carbon bonds, the authors have shown that the lattice thermal conductance is reduced without drastically affecting the electric conductance, which results in the high figure of merit $Z T=2.1$ at room temperature and $Z T>3$ at $T=700 \mathrm{~K}$. Higher value $Z T=5.8$ was even predicted for $5 \mathrm{~nm}$-wide ribbons under appropriate disorder conditions.

Another forms of layered carbon allotropes are called graphyne and graphdiyne, respectively. Graphyne is a one carbon atom thick planar sheet arranged in a crystal lattice containing both sp and $\mathrm{sp}^{2}$ hybridized states [177]. Actually, there may be a large number of possible graphynes, each with the double and triple bonds in slightly different arrangements. Though the synthesis of graphyne molecules is not strongly developed yet and only trace amounts have been fabricated in the laboratory, many theoretical works have predicted fascinating physical properties for this materials, including high electron mobility and strong mechanical properties [178]. Graphdiyne is a variant of graphyne that contains two acetylenic linkages in each unit cell rather than the one linkage as in graphyne [179]. Graphdiyne is a material softer than graphyne but its electronic properties are predicted to be exceptional [180], with a bandgap in the semiconductor range and an electron effective mass of the order of $0.08 m_{0}$, where $m_{0}$ is the electron rest mass [181]. Graphdiyne films were successfully 
synthesized on copper substrates [182] and Graphdiyne nanotube arrays were fabricated by the same group [183]. Different synthesis strategies are currently under development [184][185]. A recent review on the synthesis, properties and applications of graphyne, graphdiyne, graphane and graphone may be found in Ref. [186].

Theoretical investigations of thermoelectric performance of graphyne have been reported recently. In 2D graphyne sheets, the thermal conductance has been found to be greatly reduced compared to that of graphene in most temperature regions but larger than that of graphene at low temperature [187]. Additionally, due to the semiconductor nature of graphyne, the thermoelectric power is one order of magnitude larger than that of graphene, which results in a thermoelectric figure of merit $Z T$ reaching 0.157 at room temperature, i.e. much larger than in graphene [187]. In graphyne nanoribbons (GYNRs), it has been shown that the thermal conductance is about $40 \%$ that of graphene nanoribbons [188]. Additionally, the study of the graphyne family reveals that the thermal conductance is quite insensitive to the acetylenic linkages, but depends on the number of benzene rings. The analysis of thermoelectric performance of GYNRs and graphyne nanojunctions consisting of sections of different widths has shown that the thermoelectric figure of merit is 3-13 times larger than that in GNRs [189]. This improvement is mainly originated from the fact that the enhanced thermopower and degraded thermal conductance outweigh the reduction of electronic conductance. $Z T$ values greater than 0.6 at room temperature and 1.0 at $T=700 \mathrm{~K}$ have been predicted, which makes graphyne another potential candidate for thermoelectric applications.

\subsection{Graphene / hexagonal Boron Nitride Heterostructures}

Hexagonal Boron-Nitride (h-BN) is a wide bandgap insulator with a layered structure very similar to that of graphene but the two atoms in the unit cell are chemically non-equivalent and the lattice constant is $1.8 \%$ higher [190]. It has been shown that graphene supported on h-BN substrate has higher mobility than on any other substrate, thanks to flat and defect-free interface [191][192][193]. A mobility of $275000 \mathrm{~cm}^{2} \mathrm{~V}^{-1} \mathrm{~s}^{-1}$ at low temperatures and $125000 \mathrm{~cm}^{2} \mathrm{~V}^{-1} \mathrm{~s}^{-1}$ at room temperature, i.e. as high as that of suspended graphene, has even been reported [52]. Recently, the possibility to grow hybrid monolayers consisting of a mixture of $\mathrm{BN}$ and carbon nanodomains has been demonstrated [194][195][196][197] with the possibility to form heterostructures, which motivated new strategies of bandgap engineering and proposal of devices [196][198][199][200]. In particular, it has been suggested to introduce a finite-gap hybrid boron-nitride-carbon (BNC) region below the gate of a transistor to improve significantly the $\mathrm{I}_{\mathrm{on}} / \mathrm{I}_{\mathrm{off}}$ ratio [196], which may be also achieved thanks to a sidegated graphene/BN ribbon with electrically tunable bandgap [200]. A double-BN barrier may be used also to design a resonant tunneling device [198][199].

In terms of thermal transport, numerical simulations have shown that different types of graphene/BN heterostructures, as superlattices and embedded nanodomains, offer the possibility to tune and reduce strongly the phonon thermal conductivity [201][202][203], which is a very favorable feature to enhance the thermoelectric properties. Indeed, a very high Seebeck coefficient has been predicted in zigzag ribbons consisting of a graphene/BN superlattice, with an enhancement by a factor of 20 compared to graphene [204]. A more complete study of graphene/BN nanoribbons has shown that compared to pristine GNRs, the $Z T$ for armchair BNC ribbons is enhanced 10-20 times and can reach $Z T=0.7$ in some configurations at room temperature [205]. As expected, it is due to the combined effects of the increase in the Seebeck coefficient and the reduction in the thermal conductance outweighing the decrease in the electrical conductance. 


\subsection{Dichalcogenides}

Apart from graphene, monolayers of dichalcogenides ( $\mathrm{MX}_{2}$ compounds with $\mathrm{M}=\mathrm{Mo}, \mathrm{W}$, and $\mathrm{X}=\mathrm{S}$, $\mathrm{Se}$, or Te), atomically thin 2D semiconductors with a finite bandgap, have been the recent focus of extensive research activity [206][207]. Due to their semiconducting nature, monolayers of $\mathrm{MX}_{2}$ materials have some advantages over gapless graphene and are suitable for many electronic and photonic applications. Among this family of materials, $\mathrm{MoS}_{2}$ has been the most studied, both experimentally [208][209] and theoretically [210][211].

Within $\mathrm{MoS}_{2}$ monolayer, each Mo atom is linked to six S atoms, and each $\mathrm{S}$ atom is linked to three Mo atoms, forming a trigonal prismatic coordination configuration. It is a two-valley direct-gap $(1.8 \mathrm{eV})$ semiconductor where the conduction band and valence band edges are both located at the $\mathrm{K}$ point. In addition, compared with the nearly vanishing spin-orbit coupling in graphene, $\mathrm{MoS}_{2}$ has a stronger spin-orbit-coupling interaction, which, along with the inversion symmetry breaking, leads to coupled spin and valley physics. Magnetic properties of $\mathrm{MoS}_{2}$ have been exploited to modulate the spin-dependent tunnelling in vertical graphene/ $\mathrm{MoS}_{2}$ heterostructures [212]. Several demonstrations of single- and multilayer $\mathrm{MoS}_{2}$ and $\mathrm{MoSe}_{2}$ transistors on $\mathrm{SiO} 2$ substrate have been reported [213][214][215][216]. Thanks to the finite bandgap of $\mathrm{MoS}_{2}$, large $I_{\text {on }} / I_{\text {off }}$ ratio and good current saturation can be achieved, which is a strong advantage over graphene transistors. In spite of relatively low carrier mobility, it has even been shown that $\mathrm{MoS}_{2}$ transistors can operate with cutoff frequencies reaching $6 \mathrm{GHz}$ [217]. Additionally, integrated circuits based on $\mathrm{MoS}_{2}$ transistors were reported, demonstrating basic digital gates as an inverter, a NAND gate, a static random access memory and a five-stage ring oscillator [218]. These promising results make the investigation of $\mathrm{MoS}_{2}$ thermoelectric performance all the more stimulating.

By means of non-equilibrium molecular dynamics simulation, Liu et al. have shown that the thermal conductivity of monolayer $\mathrm{MoS}_{2}$ is only $1.35 \mathrm{~W} / \mathrm{mK}$ at room temperature, which is three orders of magnitude lower than that of graphene [219]. In contrast to the case of GNRs, the thermal conductivity of monolayer $\mathrm{MoS}_{2}$ nanoribbons (NRs) is insensitive to width and edge-type, indicating that the phonons Umklapp scattering process dominates the thermal transport in $\mathrm{MoS}_{2} \mathrm{NRs}$. Experimentally, by scanning photocurrent spectroscopy of $\mathrm{MoS}_{2}$ transistors, it has been shown that the Seebeck coefficient of single-layer $\mathrm{MoS}_{2}$ can be tuned by an external electric field between $-4 \times 10^{2}$ and $-1 \times 10^{5} \mu \mathrm{V} / \mathrm{K}$ [220]. Combining this high value of thermopower with the low thermal conductivity mentioned above, one may expect good thermoelectric performance. From first-principles DFT calculation, Huang et al. have investigated the thermoelectric performance of different kinds of dichalcogenide monolayers, including $\mathrm{MoS}_{2}, \mathrm{MoSe}_{2}, \mathrm{WS}_{2}$ and $\mathrm{WSe}_{2}$ [221]. They found that among these materials, p-type monolayer $\mathrm{MoS}_{2}$ has the highest first peak value of $Z T$ at room temperature, with $Z T>0.5$. As temperature increases, the first peak values of $Z T$ increase linearly except for monolayer n-type $\mathrm{WSe}_{2} / \mathrm{MoSe}_{2}$, and p-type $\mathrm{WS}_{2}$. At high temperature, n-type $\mathrm{WSe}_{2}$ shows an exceptional increasing rate compared to other cases, due to the electron transport contribution of an additional valley, leading to $Z T>1$ at $T=500 \mathrm{~K}$. The same authors have shown that $Z T=2.1$ is achievable in bilayer $\mathrm{WSe}_{2}$ at room temperature [222]. Hence, we can expect that by appropriate nanostructuring, this type of materials may achieve large values of thermoelectric figure of merit over a wide range of temperature.

\subsection{Phosphorene}

In the new trend toward the investigation of atomistically thin 2D materials, phosphorene, has recently emerged as a very promising one. Phosphorene is a single layer of black phosphorous arranged in a 
puckered honeycomb structure formed by $\mathrm{sp}^{3}$ hybridization of the atomic orbitals of phosporous atoms. It can be mechanically exfoliated and is stable at room temperature [223]. A single layer of phosphorene is a direct bandgap material with a strongly anisotropic dispersion in the vicinity of the gap [224]. Experimentally, it has been shown that the transport gap of multi-layer phosphorene can be tuned from $0.3 \mathrm{eV}$ to $1.0 \mathrm{eV}$ by scaling down the thickness from bulk to a single layer, which makes the on/off current ratio of field-effect transistors strongly dependent on the layer thickness [225]. Another experimental demonstration of phosphorene transistors has shown that the charge-carrier mobility is also thickness-dependent, with the highest value of about $1000 \mathrm{~cm}^{2} / \mathrm{Vs}$ for a layer thickness of $\sim 10 \mathrm{~nm}$ [226], which is significantly higher than the mobilities reached in dichalcogenide materials.

Some theoretical investigations of phosphorene thermoelectric performance have been reported recently. From first-principles calculation, Fei et al. have found that the electrical and thermal conductances exhibit strong spatial anisotropies such that their respective preferred directions of conductance are orthogonal to one another, resulting in an anisotropic thermoelectric figure of merit that is large along the armchair direction [227]. By considering a phonon relaxation time of $60 \mathrm{ps}, Z T$ has been predicted to reach more than 1 at room temperature and 2.5 at $T=500 \mathrm{~K}$. Lv et al. have shown that strain-induced band convergence in phosphorene can significantly enhance simultaneously the Seebeck coefficient and electrical conductivity, which leads to $Z T=2.1$ at room temperature in the armchair direction [228]. Zhang et al. have investigated the thermoelectric properties of phosphorene nanoribbons (PNRs) of different widths and edge configurations [229]. They demonstrated that semiconducting armchair PNRs exhibit large values of thermopower that can be further enhanced by hydrogen passivation of edge atoms. Additionally, the thermal conductivity is found to be strongly reduced depending on the ribbon width, which results in optimized value of $Z T$ as high as 6.4 at room temperature in armchair PNRs with 9 dimer lines along the ribbon width. All these results suggest that phosphorene has a strong potential for thermoelectric applications, though experimental confirmations are still needed.

\section{Conclusions}

We have reviewed experimental and theoretical results relevant to thermoelectric properties and performance of graphene, graphene nanostructures and hybrid graphene structures. Beyond the fundamental experiments on Seebeck and Nernst effects which have allowed us to understand better some specific features of the band structure and ambipolar transport in graphene, it is remarkable to observe how, starting from the poor thermoelectric performance of a 2D gapless semimetal, it is possible to tune on a wide range both the electronic and thermal conduction properties by using different strategies of nanostructuring and bandgap engineering, to such a point that high thermoelectric figures of merit become achievable. Though most of these results and predictions still need to be confirmed at the experimental level, they show clearly that graphene and other atomicallythin layered materials as dichalcogenides and phophorene are very promising for future developments of thermoelectric energy conversion at the nanoscale. Due to the high sensitivity of most physical properties of these materials to atomic defects and to the environment, a significant obstacle is obviously the fabrication of well-controlled nanostructures. However, the recent progress achieved in the bottom-up synthesis of GNRs with atomically precise edges [130] makes it realistic to envision in the near future the experimental demonstration of some fascinating results recently predicted by numerical simulations. Another important point, not addressed in this review, is the processing of good thermal contacts at the nanoscale, likely not to degrade drastically the good intrinsic performance 
of thermoelectric nanodevices. It is known as a crucial issue to optimize the working conditions of thermoelectric generators [230][231] and we can anticipate this problem to be a critical step for the transfer of graphene thermoelectrics at the application level. Actually, this research topic of graphene and 2D materials thermoelectrics is in its infancy and we have no doubts that it will be the subject of intense activities and developments in the next years.

\section{References}

[1] Goldsmid H J, Sheard A R and Wright D A 1958 The performance of bismuth telluride thermojunctions Br. J. Appl. Phys. 9 365-70

[2] Ioffe A F 1957 Semiconductor thermoelements, and Thermoelectric cooling. (London: Infosearch, ltd.)

[3] Morelli D T, Caillat T, Fleurial J-P, Borshchevsky A, Vandersande J, Chen B and Uher C 1995 Low-temperature transport properties of p-type CoSb3 Phys. Rev. B 51 9622-8

[4] Shi X, Yang J, Salvador J R, Chi M, Cho J Y, Wang H, Bai S, Yang J, Zhang W and Chen L 2011 Multiple-Filled Skutterudites: High Thermoelectric Figure of Merit through Separately Optimizing Electrical and Thermal Transports J. Am. Chem. Soc. 133 7837-46

[5] Culp S R, Simonson J W, Poon S J, Ponnambalam V, Edwards J and Tritt T M 2008 $(\mathrm{Zr}, \mathrm{Hf}) \mathrm{Co}(\mathrm{Sb}, \mathrm{Sn})$ half-Heusler phases as high-temperature $\left(>700^{\circ} \mathrm{C}\right) \mathrm{p}$-type thermoelectric materials Appl. Phys. Lett. 93022105

[6] Yan X, Joshi G, Liu W, Lan Y, Wang H, Lee S, Simonson J W, Poon S J, Tritt T M, Chen G and Ren Z F 2011 Enhanced Thermoelectric Figure of Merit of p-Type HalfHeuslers Nano Lett. 11 556-60

[7] Dresselhaus M S, Chen G, Tang M Y, Yang R G, Lee H, Wang D Z, Ren Z F, Fleurial JP and Gogna P 2007 New Directions for Low-Dimensional Thermoelectric Materials Adv. Mater. 19 1043-53

[8] Hicks L D and Dresselhaus M S 1993 Effect of quantum-well structures on the thermoelectric figure of merit Phys. Rev. B 47 12727-31

[9] Hicks L D and Dresselhaus M S 1993 Thermoelectric figure of merit of a onedimensional conductor Phys. Rev. B 47 16631-4

[10] Ziman J M 1960 Electrons and phonons: The theory of transport phenomena in solids (Oxford: Clarendon Press)

[11] Cutler M and Mott N F 1969 Observation of Anderson Localization in an Electron Gas Phys. Rev. 181 1336-40

[12] Mahan G D and Sofo J O 1996 The best thermoelectric Proc. Natl. Acad. Sci. 93 $7436-9$

[13] Hicks L D, Harman T C, Sun X and Dresselhaus M S 1996 Experimental study of the effect of quantum-well structures on the thermoelectric figure of merit Phys. Rev. B $\mathbf{5 3}$ R10493-6 
[14] Venkatasubramanian R 2000 Lattice thermal conductivity reduction and phonon localizationlike behavior in superlattice structures Phys. Rev. B 61 3091-7

[15] Chen G 1998 Thermal conductivity and ballistic-phonon transport in the cross-plane direction of superlattices Phys. Rev. B 57 14958-73

[16] Lee S-M, Cahill D G and Venkatasubramanian R 1997 Thermal conductivity of SiGe superlattices Appl. Phys. Lett. 70 2957-9

[17] Harman T C, Spears D L and Walsh M P 1999 PbTe/Te superlattice structures with enhanced thermoelectric figures of merit J. Electron. Mater. 28 L1-5

[18] Harman T C, Walsh M P, Turner G W and others 2005 Nanostructured thermoelectric materials J. Electron. Mater. 34 L19-22

[19] Caylor J C, Coonley K, Stuart J, Colpitts T and Venkatasubramanian R 2005 Enhanced thermoelectric performance in PbTe-based superlattice structures from reduction of lattice thermal conductivity Appl. Phys. Lett. 87023105

[20] Vashaee D and Shakouri A 2007 Thermionic power generation at high temperatures using SiGeSi superlattices J. Appl. Phys. 101053719

[21] Koga T, Sun X, Cronin S B and Dresselhaus M S 1998 Carrier pocket engineering to design superior thermoelectric materials using GaAs/AlAs superlattices Appl. Phys. Lett. $732950-2$

[22] Zide J M O, Vashaee D, Bian Z X, Zeng G, Bowers J E, Shakouri A and Gossard A C 2006 Demonstration of electron filtering to increase the Seebeck coefficient in In0.53Ga0.47As/n0.53Ga0.28A10.19As superlattices Phys. Rev. B 74205335

[23] Venkatasubramanian R, Siivola E, Colpitts T and O'Quinn B 2001 Thin-film thermoelectric devices with high room-temperature figures of merit Nature 413 597-602

[24] Zhao L-D, Lo S-H, Zhang Y, Sun H, Tan G, Uher C, Wolverton C, Dravid V P and Kanatzidis M G 2014 Ultralow thermal conductivity and high thermoelectric figure of merit in SnSe crystals Nature $\mathbf{5 0 8} 373-7$

[25] Vineis C J, Shakouri A, Majumdar A and Kanatzidis M G 2010 Nanostructured Thermoelectrics: Big Efficiency Gains from Small Features Adv. Mater. 22 3970-80

[26] Sassi S, Candolfi C, Vaney J-B, Ohorodniichuk V, Masschelein P, Dauscher A and Lenoir B 2014 Assessment of the thermoelectric performance of polycrystalline p-type SnSe Appl. Phys. Lett. 104212105

[27] Gudiksen M S, Lauhon L J, Wang J, Smith D C and Lieber C M 2002 Growth of nanowire superlattice structures for nanoscale photonics and electronics Nature 415617 20

[28] Hochbaum A I, Chen R, Delgado R D, Liang W, Garnett E C, Najarian M, Majumdar A and Yang P 2008 Enhanced thermoelectric performance of rough silicon nanowires Nature 451 163-7 
[29] Boukai A I, Bunimovich Y, Tahir-Kheli J, Yu J-K, Goddard Iii W A and Heath J R 2008 Silicon nanowires as efficient thermoelectric materials Nature 451 168-71

[30] Xu B, Li C, Thielemans K, Myronov M and Fobelets K 2012 Thermoelectric Performance of Si0.8Ge0.2 Nanowire Arrays IEEE Trans. Electron Devices 59 3193-8

[31] Shi L, Jiang J, Zhang G and Li B 2012 High thermoelectric figure of merit in silicongermanium superlattice structured nanowires Appl. Phys. Lett. 101233114

[32] Harman T C, Taylor P J, Walsh M P and LaForge B E 2002 Quantum Dot Superlattice Thermoelectric Materials and Devices Science 297 2229-32

[33] Hsu K F, Loo S, Guo F, Chen W, Dyck J S, Uher C, Hogan T, Polychroniadis E K and Kanatzidis M G 2004 Cubic AgPbmSbTe2+m: Bulk Thermoelectric Materials with High Figure of Merit Science 303 818-21

[34] Wang H, Li J-F, Nan C-W, Zhou M, Liu W, Zhang B-P and Kita T 2006 Highperformance $\mathrm{Ag} 0.8 \mathrm{~Pb} 18+\mathrm{xSbTe} 20$ thermoelectric bulk materials fabricated by mechanical alloying and spark plasma sintering Appl. Phys. Lett. 88092104

[35] Androulakis J, Lin C-H, Kong H-J, Uher C, Wu C-I, Hogan T, Cook B A, Caillat T, Paraskevopoulos K M and Kanatzidis M G 2007 Spinodal Decomposition and Nucleation and Growth as a Means to Bulk Nanostructured Thermoelectrics: Enhanced Performance in Pb1-xSnxTe-PbS J. Am. Chem. Soc. 129 9780-8

[36] Bao Y, Liu W L, Shamsa M, Alim K, Balandin A A and Liu J L 2005 Electrical and Thermal Conductivity of Ge/Si Quantum Dot Superlattices J. Electrochem. Soc. 152 G432-5

[37] Heremans J P, Thrush C M and Morelli D T 2004 Thermopower enhancement in lead telluride nanostructures Phys. Rev. B 70115334

[38] Heremans J P 2005 Low-dimensional thermoelectricity Acta Phys. Pol. Ser. A 108 609-34

[39] Liu W, Yan X, Chen G and Ren Z 2012 Recent advances in thermoelectric nanocomposites Nano Energy $142-56$

[40] Meyer J C, Geim A K, Katsnelson M I, Novoselov K S, Booth T J and Roth S 2007 The structure of suspended graphene sheets Nature 446 60-3

[41] Wallace P R 1947 The Band Theory of Graphite Phys. Rev. 71 622-34

[42] Castro Neto A H, Peres N M R, Novoselov K S and Geim A K 2009 The electronic properties of graphene Rev. Mod. Phys. 81 109-62

[43] Molitor F, Güttinger J, Stampfer C, Dröscher S, Jacobsen A, Ihn T and Ensslin K 2011 Electronic properties of graphene nanostructures J. Phys. Condens. Matter 23 243201

[44] Zhang Y, Tan Y-W, Stormer H L and Kim P 2005 Experimental observation of the quantum Hall effect and Berry's phase in graphene Nature 438 201-4 
[45] Novoselov K S, Geim A K, Morozov S V, Jiang D, Katsnelson M I, Grigorieva I V, Dubonos S V and Firsov A A 2005 Two-dimensional gas of massless Dirac fermions in graphene Nature 438 197-200

[46] Katsnelson M I, Novoselov K S and Geim A K 2006 Chiral tunnelling and the Klein paradox in graphene Nat. Phys. 2 620-5

[47] Adam S, Hwang E H, Galitski V M and Sarma S D 2007 A self-consistent theory for graphene transport Proc. Natl. Acad. Sci. 104 18392-7

[48] Cheianov V V, Fal'ko V I, Altshuler B L and Aleiner I L 2007 Random Resistor Network Model of Minimal Conductivity in Graphene Phys. Rev. Lett. 99176801

[49] Chen J-H, Jang C, Adam S, Fuhrer M S, Williams E D and Ishigami M 2008 Charged-impurity scattering in graphene Nat. Phys. 4 377-81

[50] Do V N and Dollfus P 2010 Modeling of metal-graphene coupling and its influence on transport properties in graphene at the charge neutrality point J. Phys. Condens. Matter 22425301

[51] Bolotin K I, Sikes K J, Jiang Z, Klima M, Fudenberg G, Hone J, Kim P and Stormer H L 2008 Ultrahigh electron mobility in suspended graphene Solid State Commun. 146 $351-5$

[52] Zomer P J, Dash S P, Tombros N and Van Wees B J 2011 A transfer technique for high mobility graphene devices on commercially available hexagonal boron nitride Appl. Phys. Lett. 99232104

[53] Cresti A, Nemec N, Biel B, Niebler G, Triozon F, Cuniberti G and Roche S 2008 Charge transport in disordered graphene-based low dimensional materials Nano Res. 1 361-94

[54] Fischetti M V, Kim J, Narayanan S, Ong Z-Y, Sachs C, Ferry D K and Aboud S J 2013 Pseudopotential-based studies of electron transport in graphene and graphene nanoribbons J. Phys. Condens. Matter 25473202

[55] Foa Torres L E F, Roche S and Charlier J-C 2014 Introduction to Graphene Based Nanomaterials From Electronic Structure to Quantum Transport (Cambridge: Cambridge University Press)

[56] Ouyang Y and Guo J 2009 A theoretical study on thermoelectric properties of graphene nanoribbons Appl. Phys. Lett. 94263107

[57] Hicks L D, Harman T C and Dresselhaus M S 1993 Use of quantum-well superlattices to obtain a high figure of merit from nonconventional thermoelectric materials Appl. Phys. Lett. 63 3230-2

[58] Farhangfar S 2011 Size-dependent thermoelectricity in nanowires J. Phys. Appl. Phys. 44125403

[59] Balandin A A, Ghosh S, Bao W, Calizo I, Teweldebrhan D, Miao F and Lau C N 2008 Superior Thermal Conductivity of Single-Layer Graphene Nano Lett. 8 902-7 
[60] Ghosh S, Bao W, Nika D L, Subrina S, Pokatilov E P, Lau C N and Balandin A A 2010 Dimensional crossover of thermal transport in few-layer graphene Nat. Mater. 9 $555-8$

[61] Balandin A A 2011 Thermal properties of graphene and nanostructured carbon materials Nat. Mater. 10 569-81

[62] Pop E, Varshney V and Roy A K 2012 Thermal properties of graphene: Fundamentals and applications MRS Bull. 37 1273-81

[63] Nika D L and Balandin A A 2012 Two-dimensional phonon transport in graphene $J$. Phys. Condens. Matter 24233203

[64] Xu Y, Li Z and Duan W 2014 Thermal and thermoelectric properties of graphene Small 10 2182-99

[65] Haskins J, Kınacı A, Sevik C, Sevinçli H, Cuniberti G and Çağın T 2011 Control of Thermal and Electronic Transport in Defect-Engineered Graphene Nanoribbons ACS Nano 5 3779-87

[66] Zuev Y, Chang W and Kim P 2009 Thermoelectric and Magnetothermoelectric Transport Measurements of Graphene Phys. Rev. Lett. 102096807

[67] Wei P, Bao W, Pu Y, Lau C and Shi J 2009 Anomalous Thermoelectric Transport of Dirac Particles in Graphene Phys. Rev. Lett. 102166808

[68] Checkelsky J and Ong N 2009 Thermopower and Nernst effect in graphene in a magnetic field Phys. Rev. B $\mathbf{8 0} 081413$

[69] Hwang E H, Rossi E and Das Sarma S 2009 Theory of thermopower in twodimensional graphene Phys. Rev. B 80235415

[70] Nam S-G, Ki D-K and Lee H-J 2010 Thermoelectric transport of massive Dirac fermions in bilayer graphene Phys. Rev. B 82245416

[71] Wu X, Hu Y, Ruan M, Madiomanana N K, Berger C and de Heer W A 2011 Thermoelectric effect in high mobility single layer epitaxial graphene Appl. Phys. Lett. 99 133102

[72] Wang D and Shi J 2011 Effect of charged impurities on the thermoelectric power of graphene near the Dirac point Phys. Rev. B 83113403

[73] Xiao N, Dong X, Song L, Liu D, Tay Y, Wu S, Li L-J, Zhao Y, Yu T, Zhang H, Huang W, Hng H H, Ajayan P M and Yan Q 2011 Enhanced Thermopower of Graphene Films with Oxygen Plasma Treatment ACS Nano 5 2749-55

[74] Wang C-R, Lu W-S, Hao L, Lee W-L, Lee T-K, Lin F, Cheng I-C and Chen J-Z 2011 Enhanced Thermoelectric Power in Dual-Gated Bilayer Graphene Phys. Rev. Lett. 107 186602 
[75] Park J, He G, Feenstra R M and Li A-P 2013 Atomic-Scale Mapping of Thermoelectric Power on Graphene: Role of Defects and Boundaries Nano Lett. 13 326973

[76] Sidorov A N, Sherehiy A, Jayasinghe R, Stallard R, Benjamin D K, Yu Q, Liu Z, Wu W, Cao H, Chen Y P, Jiang Z and Sumanasekera G U 2011 Thermoelectric power of graphene as surface charge doping indicator Appl. Phys. Lett. 99013115

[77] Dóra B and Thalmeier P 2007 Magnetotransport and thermoelectricity in Landauquantized disordered graphene Phys. Rev. B 76035402

[78] Löfwander T and Fogelström M 2007 Impurity scattering and Mott's formula in graphene Phys. Rev. B $\mathbf{7 6} 193401$

[79] Foster M S and Aleiner I L 2008 Graphene via large N: A renormalization group study Phys. Rev. B 77195413

[80] Yan X-Z, Romiah Y and Ting C 2009 Thermoelectric power of Dirac fermions in graphene Phys. Rev. B $\mathbf{8 0} 165423$

[81] Yan X-Z and Ting C S 2010 Nernst effect of Dirac fermions in graphene under a weak magnetic field Phys. Rev. B 81155457

[82] Zhu L, Ma R, Sheng L, Liu M and Sheng D-N 2010 Universal Thermoelectric Effect of Dirac Fermions in Graphene Phys. Rev. Lett. 104076804

[83] Geballe T H and Hull G W 1954 Seebeck Effect in Germanium Phys. Rev. 94 113440

[84] Fletcher R, Harris J J, Foxon C T, Tsaousidou M and Butcher P N 1994 Thermoelectric properties of a very-low-mobility two-dimensional electron gas Phys. Rev. B 50 14991-8

[85] Bao W S, Liu S Y and Lei X L 2010 Thermoelectric power in graphene J. Phys. Condens. Matter 22315502

[86] Koniakhin S V and Eidelman E D 2013 Phonon drag thermopower in graphene in equipartition regime EPL Europhys. Lett. 10337006

[87] Hao L and Lee T K 2010 Thermopower of gapped bilayer graphene Phys. Rev. B 81 165445

[88] Hao L and Lee T K 2010 Thermopower of multilayer graphene Phys. Rev. B 82 245415

[89] Ma R, Zhu L, Sheng L, Liu M and Sheng D N 2011 Thermoelectric and thermal transport in bilayer graphene systems Phys. Rev. B $\mathbf{8 4} 075420$

[90] Ma R, Sheng L, Liu M and Sheng D N 2012 Stacking-order dependence in thermoelectric transport of biased trilayer graphene Phys. Rev. B 86115414 
[91] Sharapov S G and Varlamov A A 2012 Anomalous growth of thermoelectric power in gapped graphene Phys. Rev. B $\mathbf{8 6} 035430$

[92] Lacroix D, Joulain K, Terris D and Lemonnier D 2006 Monte Carlo simulation of phonon confinement in silicon nanostructures: Application to the determination of the thermal conductivity of silicon nanowires Appl. Phys. Lett. 89103104

[93] Volz S G and Chen G 1999 Molecular dynamics simulation of thermal conductivity of silicon nanowires Appl. Phys. Lett. 75 2056-8

[94] Hu J, Ruan X and Chen Y P 2009 Thermal Conductivity and Thermal Rectification in Graphene Nanoribbons: A Molecular Dynamics Study Nano Lett. 9 2730-5

[95] Shi L-P and Xiong S-J 2009 Phonon thermal conductance of disordered graphene strips with armchair edges Phys. Lett. A 373 563-9

[96] Li W, Sevinçli H, Cuniberti G and Roche S 2010 Phonon transport in large scale carbon-based disordered materials: Implementation of an efficient order- $\mathrm{N}$ and real-space Kubo methodology Phys. Rev. B 82041410

[97] Mohr M, Maultzsch J, Dobardžić E, Reich S, Milošević I, Damnjanović M, Bosak A, Krisch M and Thomsen C 2007 Phonon dispersion of graphite by inelastic X-ray scattering Phys. Rev. B 76035439

[98] Mazzamuto F, Hung Nguyen V, Apertet Y, Caër C, Chassat C, Saint-Martin J and Dollfus P 2011 Enhanced thermoelectric properties in graphene nanoribbons by resonant tunneling of electrons Phys. Rev. B $\mathbf{8 3} 235426$

[99] Nikolić B K, Saha K K, Markussen T and Thygesen K S 2012 First-principles quantum transport modeling of thermoelectricity in single-molecule nanojunctions with graphene nanoribbon electrodes J. Comput. Electron. 11 78-92

[100] Do V N, Nguyen V H, Dollfus P and Bournel A 2008 Electronic transport and spinpolarization effects of relativisticlike particles in mesoscopic graphene structures J. Appl. Phys. 104063708

[101] Ouyang Y, Campbell P and Guo J 2008 Analysis of ballistic monolayer and bilayer graphene field-effect transistors Appl. Phys. Lett. 92063120

[102] Nguyen V H, Bournel A, Nguyen V L and Dollfus P 2009 Resonant tunneling and negative transconductance in single barrier bilayer graphene structure Appl. Phys. Lett. 95 232115

[103] Fiori G and Iannaccone G 2007 Simulation of Graphene Nanoribbon Field-Effect Transistors IEEE Electron Device Lett. 28 760-2

[104] Liang G, Neophytou N, Lundstrom M S and Nikonov D E 2007 Ballistic graphene nanoribbon metal-oxide-semiconductor field-effect transistors: A full real-space quantum transport simulation J. Appl. Phys. 102054307

[105] Zhao P, Chauhan J and Guo J 2009 Computational Study of Tunneling Transistor Based on Graphene Nanoribbon Nano Lett. 9 684-8 
[106] Do V N and Dollfus P 2010 Negative differential resistance in zigzag-edge graphene nanoribbon junctions J. Appl. Phys. 107063705

[107] Nguyen V H, Mazzamuto F, Saint-Martin J, Bournel A and Dollfus P 2012 Graphene nanomesh-based devices exhibiting a strong negative differential conductance effect Nanotechnology 23289502

[108] Sevinçli H and Cuniberti G 2010 Enhanced thermoelectric figure of merit in edgedisordered zigzag graphene nanoribbons Phys. Rev. B 81113401

[109] Mazzamuto F, Saint-Martin J, Nguyen V H, Chassat C and Dollfus P 2012 Thermoelectric performance of disordered and nanostructured graphene ribbons using Green's function method J. Comput. Electron. 11 67-77

[110] Karamitaheri H, Pourfath M, Faez R and Kosina H 2011 Geometrical effects on the thermoelectric properties of ballistic graphene antidot lattices J. Appl. Phys. 110054506

[111] Mazzamuto F, Saint-Martin J, Valentin A, Chassat C and Dollfus P 2011 Edge shape effect on vibrational modes in graphene nanoribbons: A numerical study J. Appl. Phys. 109064516

[112] Novoselov K S, Geim A K, Morozov S V, Jiang D, Zhang Y, Dubonos S V, Grigorieva I V and Firsov A A 2004 Electric Field Effect in Atomically Thin Carbon Films Science 306 666-9

[113] Ghosh S, Calizo I, Teweldebrhan D, Pokatilov E P, Nika D L, Balandin A A, Bao W, Miao F and Lau C N 2008 Extremely high thermal conductivity of graphene: Prospects for thermal management applications in nanoelectronic circuits Appl. Phys. Lett. 92 151911

[114] Sivan U and Imry Y 1986 Multichannel Landauer formula for thermoelectric transport with application to thermopower near the mobility edge Phys. Rev. B 33 551-8

[115] Datta S 2000 Nanoscale device modeling: the Green's function method Superlattices Microstruct. 28 253-78

[116] Anantram M P, Lundstrom M S and Nikonov D E 2008 Modeling of Nanoscale Devices Proc. IEEE 96 1511-50

[117] Dragoman D and Dragoman M 2007 Giant thermoelectric effect in graphene Appl. Phys. Lett. 91203116

[118] Cheng M 2012 Large thermoelectric effect in graphene superlattices Phys. E LowDimens. Syst. Nanostructures 46 189-92

[119] Xing Y, Sun Q and Wang J 2009 Nernst and Seebeck effects in a graphene nanoribbon Phys. Rev. B $\mathbf{8 0} 235411$

[120] Divari P C and Kliros G S 2010 Modeling the thermopower of ballistic graphene ribbons Phys. E Low-Dimens. Syst. Nanostructures 42 2431-5 
[121] Nissimagoudar A S and Sankeshwar N S 2013 Electronic thermal conductivity and thermopower of armchair graphene nanoribbons Carbon 52 201-8

[122] Kliros G S and Divari P C 2012 Thermoelectric properties of gated graphene ribbons in the ballistic regime Superlattices Microstruct. 52 221-33

[123] Yeo P S E, Sullivan M B, Loh K P and Gan C K 2013 First-principles study of the thermoelectric properties of strained graphene nanoribbons J. Mater. Chem. A 110762

[124] Chen Y, Jayasekera T, Calzolari A, Kim K W and Buongiorno Nardelli M 2010 Thermoelectric properties of graphene nanoribbons, junctions and superlattices J. Phys. Condens. Matter 22372202

[125] Xie Z-X, Tang L-M, Pan C-N, Li K-M, Chen K-Q and Duan W 2012 Enhancement of thermoelectric properties in graphene nanoribbons modulated with stub structures Appl. Phys. Lett. 100073105

[126] Pan C-N, Xie Z-X, Tang L-M and Chen K-Q 2012 Ballistic thermoelectric properties in graphene-nanoribbon-based heterojunctions Appl. Phys. Lett. 101103115

[127] Larsson M, Antonyuk V B, Mal'shukov A G and Chao K A 2003 Thermopower anomaly in multiple barrier structures Phys. Rev. B 68233302

[128] Nguyen V H, Mazzamuto F, Saint-Martin J, Bournel A and Dollfus P 2011 Giant effect of negative differential conductance in graphene nanoribbon pn hetero-junctions Appl. Phys. Lett. 99042105

[129] Cai J, Ruffieux P, Jaafar R, Bieri M, Braun T, Blankenburg S, Muoth M, Seitsonen A P, Saleh M, Feng X, Müllen K and Fasel R 2010 Atomically precise bottom-up fabrication of graphene nanoribbons Nature 466 470-3

[130] Cai J, Pignedoli C A, Talirz L, Ruffieux P, Söde H, Liang L, Meunier V, Berger R, Li R, Feng X, Müllen K and Fasel R 2014 Graphene nanoribbon heterojunctions Nat. Nanotechnol. 9 896-900

[131] Huang W, Wang J-S and Liang G 2011 Theoretical study on thermoelectric properties of kinked graphene nanoribbons Phys. Rev. B 84045410

[132] Liang L, Cruz-Silva E, Girão E C and Meunier V 2012 Enhanced thermoelectric figure of merit in assembled graphene nanoribbons Phys. Rev. B 86

[133] Liang L and Meunier V 2013 Electronic and thermoelectric properties of assembled graphene nanoribbons with elastic strain and structural dislocation Appl. Phys. Lett. 102 143101

[134] Sevinçli H, Sevik C, Çağın T and Cuniberti G 2013 A bottom-up route to enhance thermoelectric figures of merit in graphene nanoribbons Sci. Rep. 31228

[135] Karamitaheri H, Neophytou N, Pourfath M, Faez R and Kosina H 2012 Engineering enhanced thermoelectric properties in zigzag graphene nanoribbons J. Appl. Phys. 111 054501 
[136] Saha K K, Markussen T, Thygesen K S and Nikolić B K 2011 Multiterminal singlemolecule-graphene-nanoribbon junctions with the thermoelectric figure of merit optimized via evanescent mode transport and gate voltage Phys. Rev. B 84

[137] Yan Y, Liang Q-F, Zhao H and Wu C-Q 2012 Thermoelectric properties of hexagonal graphene quantum dots Phys. Lett. A 376 1154-8

[138] Yan Y, Liang Q-F, Zhao H, Wu C-Q and Li B 2012 Thermoelectric properties of onedimensional graphene antidot arrays Phys. Lett. A 376 2425-9

[139] Chang P-H and Nikolić B K 2012 Edge currents and nanopore arrays in zigzag and chiral graphene nanoribbons as a route toward high-ZT thermoelectrics Phys. Rev. B $\mathbf{8 6}$ 041406

[140] Chang P-H, Bahramy M S, Nagaosa N and Nikolić B K 2014 Giant Thermoelectric Effect in Graphene-Based Topological Insulators with Heavy Adatoms and Nanopores Nano Lett. 14 3779-84

[141] Bai J, Zhong X, Jiang S, Huang Y and Duan X 2010 Graphene nanomesh Nat. Nanotechnol. 5 190-4

[142] Liang X, Jung Y-S, Wu S, Ismach A, Olynick D L, Cabrini S and Bokor J 2010 Formation of Bandgap and Subbands in Graphene Nanomeshes with Sub-10 nm Ribbon Width Fabricated via Nanoimprint Lithography Nano Lett. 10 2454-60

[143] Safron N S, Brewer A S and Arnold M S 2011 Semiconducting Two-Dimensional Graphene Nanoconstriction Arrays Small 7 492-8

[144] Jung I, Jang H Y and Park S 2013 Direct growth of graphene nanomesh using a Au nano-network as a metal catalyst via chemical vapor deposition Appl. Phys. Lett. 103 023105

[145] Pedersen T, Flindt C, Pedersen J, Mortensen N, Jauho A-P and Pedersen K 2008 Graphene Antidot Lattices: Designed Defects and Spin Qubits Phys. Rev. Lett. 100 136804

[146] Jippo H, Ohfuchi M and Kaneta C 2011 Theoretical study on electron transport properties of graphene sheets with two- and one-dimensional periodic nanoholes Phys. Rev. B 84075467

[147] Oswald W and Wu Z 2012 Energy gaps in graphene nanomeshes Phys. Rev. B 85 115431

[148] Dvorak M, Oswald W and Wu Z 2013 Bandgap Opening by Patterning Graphene Sci. Rep. 32289

[149] Nguyen V H, Nguyen M C, Nguyen H-V and Dollfus P 2013 Disorder effects on electronic bandgap and transport in graphene-nanomesh-based structures $J$. Appl. Phys. 113013702

[150] Yang J, Ma M, Li L, Zhang Y, Huang W and Dong X 2014 Graphene nanomesh: new versatile materials Nanoscale 6 13301-13 
[151] Pedersen J G, Gunst T, Markussen T and Pedersen T G 2012 Graphene antidot lattice waveguides Phys. Rev. B $\mathbf{8 6} 245410$

[152] Nikitin A Y, Guinea F and Martin-Moreno L 2012 Resonant plasmonic effects in periodic graphene antidot arrays Appl. Phys. Lett. 101151119

[153] Berrada S, Nguyen V H, Querlioz D, Saint-Martin J, Alarcón A, Chassat C, Bournel A and Dollfus P 2013 Graphene nanomesh transistor with high on/off ratio and good saturation behavior Appl. Phys. Lett. 103183509

[154] Gunst T, Markussen T, Jauho A-P and Brandbyge M 2011 Thermoelectric properties of finite graphene antidot lattices Phys. Rev. B 84155449

[155] Nguyen V H, Nguyen M C, Nguyen H-V, Saint-Martin J and Dollfus P 2014 Enhanced thermoelectric figure of merit in vertical graphene junctions Appl. Phys. Lett.

105133105

[156] Uchida K, Ota T, Harii K, Takahashi S, Maekawa S, Fujikawa Y and Saitoh E 2010 Spin-Seebeck effects in films Solid State Commun. 150 524-8

[157] Bauer G E W, Saitoh E and van Wees B J 2012 Spin caloritronics Nat. Mater. 11 391-9

[158] Uchida K, Takahashi S, Harii K, Ieda J, Koshibae W, Ando K, Maekawa S and Saitoh E 2008 Observation of the spin Seebeck effect Nature 455 778-81

[159] Uchida K, Xiao J, Adachi H, Ohe J, Takahashi S, Ieda J, Ota T, Kajiwara Y, Umezawa H, Kawai H, Bauer G E W, Maekawa S and Saitoh E 2010 Spin Seebeck insulator Nat. Mater. 9 894-7

[160] Jaworski C M, Yang J, Mack S, Awschalom D D, Heremans J P and Myers R C 2010 Observation of the spin-Seebeck effect in a ferromagnetic semiconductor Nat. Mater. 9 898-903

[161] Zeng M, Feng Y and Liang G 2011 Graphene-based Spin Caloritronics Nano Lett. 11 1369-73

[162] Cheng S 2012 Spin thermopower and thermoconductance in a ferromagnetic graphene nanoribbon J. Phys. Condens. Matter 24385302

[163] Zhao Z, Zhai X and Jin G 2012 Thermally driven spin transport through a transversebiased zigzag-edge graphene nanoribbon J. Phys. Condens. Matter 24095302

[164] Ni Y, Yao K, Fu H, Gao G, Zhu S and Wang S 2013 Spin Seebeck Effect and Thermal Colossal Magnetoresistance in Graphene Nanoribbon Heterojunction Sci. Rep. 3 1380

[165] Torres A, Lima M P, Fazzio A and da Silva A J 2014 Spin caloritronics in graphene with Mn Appl. Phys. Lett. 104072412

[166] Zeng M, Huang W and Liang G 2013 Spin-dependent thermoelectric effects in graphene-based spin valves Nanoscale 5 200-8 
[167] Wierzbicki M, Swirkowicz R and Barnaś J 2013 Giant spin thermoelectric efficiency in ferromagnetic graphene nanoribbons with antidots Phys. Rev. B $\mathbf{8 8} 235434$

[168] Sofo J O, Chaudhari A S and Barber G D 2007 Graphane: A two-dimensional hydrocarbon Phys. Rev. B 75153401

[169] Roman T, Diño W A, Nakanishi H, Kasai H, Sugimoto T and Tange K 2007 Hydrogen pairing on graphene Carbon 45 218-20

[170] Elias D C, Nair R R, Mohiuddin T M G, Morozov S V, Blake P, Halsall M P, Ferrari A C, Boukhvalov D W, Katsnelson M I, Geim A K and Novoselov K S 2009 Control of Graphene's Properties by Reversible Hydrogenation: Evidence for Graphane Science $\mathbf{3 2 3}$ 610-3

[171] Zhou J, Wang Q, Sun Q, Chen X S, Kawazoe Y and Jena P 2009 Ferromagnetism in Semihydrogenated Graphene Sheet Nano Lett. $93867-70$

[172] Balog R, Jørgensen B, Nilsson L, Andersen M, Rienks E, Bianchi M, Fanetti M, Lægsgaard E, Baraldi A, Lizzit S, Sljivancanin Z, Besenbacher F, Hammer B, Pedersen T G, Hofmann P and Hornekær L 2010 Bandgap opening in graphene induced by patterned hydrogen adsorption Nat. Mater. 9 315-9

[173] Jaiswal M, Yi Xuan Lim C H, Bao Q, Toh C T, Loh K P and Özyilmaz B 2011 Controlled Hydrogenation of Graphene Sheets and Nanoribbons ACS Nano 5 888-96

[174] Wojtaszek M, Tombros N, Caretta A, Loosdrecht P H M van and Wees B J van 2011 A road to hydrogenating graphene by a reactive ion etching plasma J. Appl. Phys. 110 063715

[175] Liu B, Reddy C D, Jiang J, Baimova J A, Dmitriev S V, Nazarov A A and Zhou K 2012 Morphology and in-plane thermal conductivity of hybrid graphene sheets Appl. Phys. Lett. 101211909

[176] Ni X, Liang G, Wang J-S and Li B 2009 Disorder enhances thermoelectric figure of merit in armchair graphane nanoribbons Appl. Phys. Lett. 95192114

[177] Baughman R H, Eckhardt H and Kertesz M 1987 Structure-property predictions for new planar forms of carbon: Layered phases containing sp2 and sp atoms J. Chem. Phys. 87 6687-99

[178] Cranford S W and Buehler M J 2011 Mechanical properties of graphyne Carbon 49 4111-21

[179] Haley M M 2008 Synthesis and properties of annulenic subunits of graphyne and graphdiyne nanoarchitectures Pure Appl. Chem. 80 519-32

[180] Long M, Tang L, Wang D, Li Y and Shuai Z 2011 Electronic Structure and Carrier Mobility in Graphdiyne Sheet and Nanoribbons: Theoretical Predictions ACS Nano 5 2593-600

[181] Pei Y 2012 Mechanical properties of graphdiyne sheet Phys. B Condens. Matter 407 4436-9 
[182] Li G, Li Y, Liu H, Guo Y, Li Y and Zhu D 2010 Architecture of graphdiyne nanoscale films Chem. Commun. 46 3256-8

[183] Li G, Li Y, Qian X, Liu H, Lin H, Chen N and Li Y 2011 Construction of Tubular Molecule Aggregations of Graphdiyne for Highly Efficient Field Emission J. Phys. Chem. C 115 2611-5

[184] Wang S, Yi L, Halpert J E, Lai X, Liu Y, Cao H, Yu R, Wang D and Li Y 2012 A Novel and Highly Efficient Photocatalyst Based on P25-Graphdiyne Nanocomposite Small 8 265-71

[185] Cranford S W and Buehler M J 2012 Selective hydrogen purification through graphdiyne under ambient temperature and pressure Nanoscale 4 4587-93

[186] Peng Q, Crean J, Han L, Liu S, Wen X, De S and Dearden A 2014 New materials graphyne, graphdiyne, graphone, and graphane: review of properties, synthesis, and application in nanotechnology Nanotechnol. Sci. Appl. 7 1-29

[187] Wang X-M, Mo D-C and Lu S-S 2013 On the thermoelectric transport properties of graphyne by the first-principles method J. Chem. Phys. 138204704

[188] Ouyang T, Chen Y, Liu L-M, Xie Y, Wei X and Zhong J 2012 Thermal transport in graphyne nanoribbons Phys. Rev. B 85235436

[189] Ouyang T, Xiao H, Xie Y, Wei X, Chen Y and Zhong J 2013 Thermoelectric properties of gamma-graphyne nanoribbons and nanojunctions J. Appl. Phys. 114073710

[190] Liu L, Feng Y P and Shen Z X 2003 Structural and electronic properties of h-BN Phys. Rev. B $\mathbf{6 8} 104102$

[191] Dean C R, Young A F, Meric I, Lee C, Wang L, Sorgenfrei S, Watanabe K, Taniguchi T, Kim P, Shepard K L and Hone J 2010 Boron nitride substrates for highquality graphene electronics Nat. Nanotechnol. 5 722-6

[192] Xue J, Sanchez-Yamagishi J, Bulmash D, Jacquod P, Deshpande A, Watanabe K, Taniguchi T, Jarillo-Herrero P and LeRoy B J 2011 Scanning tunnelling microscopy and spectroscopy of ultra-flat graphene on hexagonal boron nitride Nat. Mater. 10 282-5

[193] Decker R, Wang Y, Brar V W, Regan W, Tsai H-Z, Wu Q, Gannett W, Zettl A and Crommie M F 2011 Local Electronic Properties of Graphene on a BN Substrate via Scanning Tunneling Microscopy Nano Lett. 11 2291-5

[194] Ci L, Song L, Jin C, Jariwala D, Wu D, Li Y, Srivastava A, Wang Z F, Storr K, Balicas L, Liu F and Ajayan P M 2010 Atomic layers of hybridized boron nitride and graphene domains Nat. Mater. 9 430-5

[195] Sutter P, Cortes R, Lahiri J and Sutter E 2012 Interface Formation in Monolayer Graphene-Boron Nitride Heterostructures Nano Lett. 12 4869-74

[196] Liu Z, Ma L, Shi G, Zhou W, Gong Y, Lei S, Yang X, Zhang J, Yu J, Hackenberg K P, Babakhani A, Idrobo J-C, Vajtai R, Lou J and Ajayan P M 2013 In-plane 
heterostructures of graphene and hexagonal boron nitride with controlled domain sizes Nat. Nanotechnol. 8 119-24

[197] Liu L, Park J, Siegel D A, McCarty K F, Clark K W, Deng W, Basile L, Idrobo J C, Li A-P and Gu G 2014 Heteroepitaxial Growth of Two-Dimensional Hexagonal Boron Nitride Templated by Graphene Edges Science 343 163-7

[198] Nguyen V H, Mazzamuto F, Bournel A and Dollfus P 2012 Resonant tunnelling diodes based on graphene/h-BN heterostructure J. Phys. Appl. Phys. 45325104

[199] Fiori G, Betti A, Bruzzone S and Iannaccone G 2012 Lateral Graphene-hBCN Heterostructures as a Platform for Fully Two-Dimensional Transistors ACS Nano 6 26428

[200] Tran V-T, Saint-Martin J and Dollfus P 2014 Large on/off current ratio in hybrid graphene/BN nanoribbons by transverse electric field-induced control of bandgap Appl. Phys. Lett. 105073114

[201] Jiang J-W, Wang J-S and Wang B-S 2011 Minimum thermal conductance in graphene and boron nitride superlattice Appl. Phys. Lett. 99043109

[202] Sevinçli H, Li W, Mingo N, Cuniberti G and Roche S 2011 Effects of domains in phonon conduction through hybrid boron nitride and graphene sheets Phys. Rev. B 84 205444

[203] Kınacı A, Haskins J B, Sevik C and Çağın T 2012 Thermal conductivity of BN-C nanostructures Phys. Rev. B 86115410

[204] Yokomizo Y and Nakamura J 2013 Giant Seebeck coefficient of the graphene/h-BN superlattices Appl. Phys. Lett. 103113901

[205] Yang K, Chen Y, D’Agosta R, Xie Y, Zhong J and Rubio A 2012 Enhanced thermoelectric properties in hybrid graphene/boron nitride nanoribbons Phys. Rev. B 86 045425

[206] Wang Q H, Kalantar-Zadeh K, Kis A, Coleman J N and Strano M S 2012 Electronics and optoelectronics of two-dimensional transition metal dichalcogenides Nat. Nanotechnol. 7 699-712

[207] Kang J, Tongay S, Zhou J, Li J and Wu J 2013 Band offsets and heterostructures of two-dimensional semiconductors Appl. Phys. Lett. 102012111

[208] Mak K F, Lee C, Hone J, Shan J and Heinz T F 2010 Atomically Thin MoS2: A New Direct-Gap Semiconductor Phys. Rev. Lett. 105136805

[209] Yu Y, Li C, Liu Y, Su L, Zhang Y and Cao L 2013 Controlled Scalable Synthesis of Uniform, High-Quality Monolayer and Few-layer MoS2 Films Sci. Rep. 31866

[210] Kaasbjerg K, Thygesen K S and Jacobsen K W 2012 Phonon-limited mobility in ntype single-layer MoS2 from first principles Phys. Rev. B 85115317 
[211] Kormányos A, Zólyomi V, Drummond N D, Rakyta P, Burkard G and Fal'ko V I 2013 Monolayer MoS2: Trigonal warping, the $\Gamma$ valley, and spin-orbit coupling effects Phys. Rev. B 88045416

[212] Myoung N, Seo K, Lee S J and Ihm G 2013 Large Current Modulation and SpinDependent Tunneling of Vertical Graphene/ $\mathrm{MoS}_{2}$ Heterostructures ACS Nano 7 7021-7

[213] Radisavljevic B, Radenovic A, Brivio J, Giacometti V and Kis A 2011 Single-layer MoS2 transistors Nat. Nanotechnol. 6 147-50

[214] Hwang W S, Remskar M, Yan R, Kosel T, Park J K, Cho B J, Haensch W, Xing H (Grace), Seabaugh A and Jena D 2013 Comparative study of chemically synthesized and exfoliated multilayer MoS2 field-effect transistors Appl. Phys. Lett. 102043116

[215] Das S, Chen H-Y, Penumatcha A V and Appenzeller J 2013 High Performance Multilayer $\mathrm{MoS}_{2}$ Transistors with Scandium Contacts Nano Lett. 13 100-5

[216] Larentis S, Fallahazad B and Tutuc E 2012 Field-effect transistors and intrinsic mobility in ultra-thin MoSe2 layers Appl. Phys. Lett. 101223104

[217] Krasnozhon D, Lembke D, Nyffeler C, Leblebici Y and Kis A 2014 MoS2 Transistors Operating at Gigahertz Frequencies Nano Lett. 14 5905-11

[218] Wang H, Yu L, Lee Y-H, Shi Y, Hsu A, Chin M L, Li L-J, Dubey M, Kong J and Palacios T 2012 Integrated Circuits Based on Bilayer MoS2 Transistors Nano Lett. 12 4674-80

[219] Liu X, Zhang G, Pei Q-X and Zhang Y-W 2013 Phonon thermal conductivity of monolayer MoS2 sheet and nanoribbons Appl. Phys. Lett. 103133113

[220] Buscema M, Barkelid M, Zwiller V, van der Zant H S J, Steele G A and CastellanosGomez A 2013 Large and Tunable Photothermoelectric Effect in Single-Layer $\mathrm{MoS}_{2}$ Nano Lett. 13 358-63

[221] Huang W, Da H and Liang G 2013 Thermoelectric performance of MX2 ( $\mathrm{M}=\mathrm{Mo}$,W; $\mathrm{X}=\mathrm{S}, \mathrm{Se}$ ) monolayers J. Appl. Phys. 113104304

[222] Huang W, Luo X, Gan C K, Quek S Y and Liang G 2014 Theoretical study of thermoelectric properties of few-layer MoS2 and WSe2 Phys. Chem. Chem. Phys. 16 $10866-74$

[223] Liu H, Neal A T, Zhu Z, Luo Z, Xu X, Tománek D and Ye P D 2014 Phosphorene: An Unexplored 2D Semiconductor with a High Hole Mobility ACS Nano 8 4033-41

[224] Rodin A S, Carvalho A and Castro Neto A H 2014 Strain-Induced Gap Modification in Black Phosphorus Phys. Rev. Lett. 112176801

[225] Das S, Zhang W, Demarteau M, Hoffmann A, Dubey M and Roelofs A 2014 Tunable Transport Gap in Phosphorene Nano Lett. 14 5733-9

[226] Li L, Yu Y, Ye G J, Ge Q, Ou X, Wu H, Feng D, Chen X H and Zhang Y 2014 Black phosphorus field-effect transistors Nat. Nanotechnol. $9372-7$ 
[227] Fei R, Faghaninia A, Soklaski R, Yan J-A, Lo C and Yang L 2014 Enhanced Thermoelectric Efficiency via Orthogonal Electrical and Thermal Conductances in Phosphorene Nano Lett. 14 6393-9

[228] Lv H Y, Lu W J, Shao D F and Sun Y P 2014 Enhanced thermoelectric performance of phosphorene by strain-induced band convergence Phys. Rev. B 90085433

[229] Zhang J, Liu H J, Cheng L, Wei J, Liang J H, Fan D D, Shi J, Tang X F and Zhang Q J 2014 Phosphorene nanoribbon as a promising candidate for thermoelectric applications Sci. Rep. 46452

[230] Apertet Y, Ouerdane H, Glavatskaya O, Goupil C and Lecoeur P 2012 Optimal working conditions for thermoelectric generators with realistic thermal coupling $E P L$ Europhys. Lett. 9728001

[231] Grosse K L, Bae M-H, Lian F, Pop E and King W P 2011 Nanoscale Joule heating, Peltier cooling and current crowding at graphene-metal contacts Nat. Nanotechnol. 6 287-90 


\section{Figure Captions}

Figure 1. (a) Electrical conductivity and (b) Thermoelectric power (TEP) of a graphene sample as a function of $V_{g}$ for $T=300 \mathrm{~K}$ (square), $150 \mathrm{~K}$ (circle), $80 \mathrm{~K}$ (up triangle), $40 \mathrm{~K}$ (down triangle), and 10 $\mathrm{K}$ (diamond). Upper inset: Scanning electron microscopy image of a typical device, the scale bar is 2 $\mu \mathrm{m}$. Lower inset: Thermopower values taken at $V_{g}=-30 \mathrm{~V}$ (square) and - $5 \mathrm{~V}$ (circle). Dashed lines are linear fits to the data. Reproduced with permission [66]. Copyright 2009, American Physical Society.

Figure 2. Thermoelectric measurements in bilayer graphene. (a) Two-terminal resistance $R$ and (b) thermopower as a function of the backgate voltage $V_{B G}$ in zero magnetic field and at various temperatures: $T=30,50,70,140,170$, and $250 \mathrm{~K}$. Inset: optical image of a typical device. Size of the scalebar is $30 \mathrm{~nm}$. Reproduced with permission [70]. Copyright 2010, American Physical Society.

Figure 3. . Linear- $T$ dependence coefficient $S / T$ of thermoelectric power as a function of the electron doping concentration $\delta$. The present calculations (red solid line with circles for renormalized $\mu$ and the blue dashed line with squares for $\mu=E_{F}$ ) are compared with the experimental data in Ref. [66] (circles at $T=150 \mathrm{~K}$ and squares at $T=300 \mathrm{~K}$ ), Ref. [67] (hexagons at $T=255 \mathrm{~K}$ ), and Ref. [68] (up triangles at $T=160 \mathrm{~K}$ and down triangles at $T=280 \mathrm{~K}$ ). Reproduced with permission [80]. Copyright 2009, American Physical Society.

Figure 4. Thermopower $S_{x x}$ and Nernst signal $S_{x y}$ as functions of the Fermi energy in bilayer graphene and at different temperatures. The system size is taken to be $N=96 \times 48 \times 2$, with magnetic flux $\varphi=2 \pi / 48$, and disorder strength $r_{s}=0.3$ (uniformly distributed positively and negatively charged impurities are considered within this strength) with $W_{L / \gamma_{0}}=0.0376$. Reproduced with permission [89]. Copyright 2011, American Physical Society.

Figure 5. (a) MGNR consisting of alternating 8-AGNR and 20-ZGNR sections, and local density of states at energy $E=0.05 \mathrm{eV}$. Red and blue areas correspond to high and low DOS, respectively. (b) Phonon contribution to the thermal conductance as a function of temperature in MGNR and 8-AGNR. (c) Electronic conductance and (d) figure of merit $Z T$ as a function of chemical potential in both GNRs at room temperature. Further details in Ref. [98].

Figure 6. Structural aspects and isotope distribution types of bottom-up fabricated graphene nanoribbons (GNRs). (a) Structures of precursors and corresponding straight and chevron type graphene nanoribbons, s-GNR and c-GNR. (b) Heavy isotopes can be distributed at the atomic or precursor level. Grey, 12C; black, 14C; white H. Reproduced with permission [134]. Copyright 2013, Nature Publishing Group. 
Figure 7. Maximum $Z T$ as a function of length at different temperatures in structures schematized in Fig. 6. Maximum $Z T$ achievable when Anderson type disorder is introduced in the electronic Hamiltonian. The variation of onsite energies are set equal to the temperature, $\sigma=k_{B} T$. Solid lines represent hole-like transport while the dashed lines are for electron-like particles. The $Z T_{\max }$ values shown are realized inside the band gap, i.e $|\mu|<0.75 \mathrm{eV}$ with respect to the mid-gap, except for the dotted curve $(T=300 \mathrm{~K})$ when $|\mu|>1$. Reproduced with permission [134]. Copyright 2013, Nature Publishing Group.

Figure 8. Schematic view of (a) ZGNR|18-annulene| ZGNR and (b) ZGNR|C10|ZGNR singlemolecule nanojunctions. The contact between the source and the drain 8-ZGNR (consisting of eight zigzag chains) metallic electrodes and molecules is made via five-membered rings of carbon atoms (dark blue). The hydrogen atoms (light yellow) are included to passivate the edge carbon atoms in the nanoribbon or dangling bonds in the molecules. The distance between ZGNR electrodes is $d=1.29 \mathrm{~nm}$ for the device in panel (a) and $d=1.59 \mathrm{~nm}$ for the device in panel (b). Reproduced with permission [99]. Copyright 2012, Springer.

Figure 9. A typical H-passivated GNM system. Here the big cyan (small blue) spheres represent C (H) atoms, and $\mathrm{W}$ denotes the neck width along the zigzag and armchair directions. Reproduced with permission [147]. Copyright 2012, American Physical Society. 